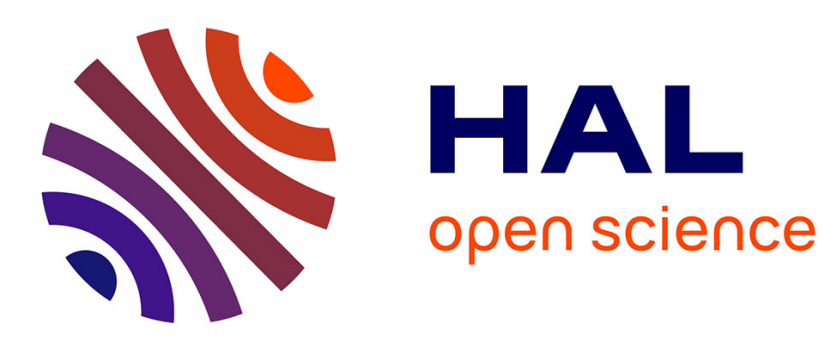

\title{
The effect of trading futures on short sale constraints
}

Robert Jarrow, Philip Protter, Sergio Pulido

\section{To cite this version:}

Robert Jarrow, Philip Protter, Sergio Pulido. The effect of trading futures on short sale constraints. Mathematical Finance, 2015, 25 (2), pp.311-338. 10.1111/mafi.12013 . hal-02265269

\section{HAL Id: hal-02265269 \\ https://hal.science/hal-02265269}

Submitted on 9 Aug 2019

HAL is a multi-disciplinary open access archive for the deposit and dissemination of scientific research documents, whether they are published or not. The documents may come from teaching and research institutions in France or abroad, or from public or private research centers.
L'archive ouverte pluridisciplinaire HAL, est destinée au dépôt et à la diffusion de documents scientifiques de niveau recherche, publiés ou non, émanant des établissements d'enseignement et de recherche français ou étrangers, des laboratoires publics ou privés. 


\title{
The Effect of Trading Futures on Short Sale Constraints*
}

\author{
Robert Jarrow $^{\dagger} \quad$ Philip Protter ${ }^{\Phi} \quad$ Sergio Pulido \\ January 5, 2013
}

\begin{abstract}
It is commonly believed that the trading of futures on a commodity enables the market to overcome short selling constraints on the spot commodity itself. This belief is embedded in the notion that trading strategies involving futures contracts enable traders to replicate the payoffs as if they were short the spot commodity. The purpose of this paper is to investigate this common belief in a general arbitrage-free semimartingale financial model with trading in futures and a short selling prohibition on the spot commodity. We show via various examples that, in general, this common belief is incorrect. Furthermore, we provide a set of sufficient conditions, albeit very restrictive, under which the common belief is true.

KEY WORDS: Short sale constraints, futures contracts, futures prices, complete markets, martingale representation, supermartingale measures, overpricing hypothesis, bubbles
\end{abstract}

\section{INTRODUCTION}

In the recent financial crisis, short sales bans and restrictions were used by regulators to stabilize declining prices (see Beber and Pagano (2011), Boehmer et al. (2011)). For the same reasons, in most third world emerging markets, short selling is either not allowed or is not possible due to inadequate financial infrastructures (see Bris et al. (2007), Charoenrook and Daouk (2005)). Even

${ }^{*}$ We would like to thank Professor Walter Schachermayer for providing the main ideas that led to Example 6.9. We would also like to thank the anonymous referees for their careful reviews of the manuscript.

†Johnson Graduate School of Management, Cornell University, Ithaca, NY, 14853 and Kamakura Corporation.

${ }^{\ddagger}$ Statistics Department, Columbia University, New York, NY, 10027.

$\S$ Supported in part by NSF grant DMS-0906995

`Department of Mathematical Sciences, Carnegie Mellon University, Pittsburgh, PA, 15213-3890 
in well developed markets, for many commodities, short selling is impossible because the underlying asset cannot be borrowed, e.g. residential homes. When an asset cannot be shorted, it is often believed that the market may be "overpriced" because the market value does not reflect negative sentiments. This is called the overpricing hypothesis (see for instance Miller (1977), Chapter 7 of Fabozzi (2004), Boulton and Braga Alves (2010) and Brown and Rogers (2012)). Such an overpricing can lead to an inefficient allocation of investments within the economy. Consequently, the inability to short an asset is often used as one justification for introducing the trading of futures contracts on that asset (see for instance Chapter 3 in Duffie (1989)). The common belief is that trading futures contracts on an asset enables the market effectively to short the spot asset, thereby completing the market. The purpose of this paper is to investigate the validity of this belief in a general arbitrage-free semimartingale financial model with trading in futures and short sale prohibitions on the spot asset.

We show via various examples that, in general, this common belief is incorrect: one cannot use futures to replicate the payoffs of being short an asset. Furthermore, we provide a set of sufficient conditions, albeit very restrictive, where this common belief is true. These results are based on the version of the Fundamental Theorem of Asset Pricing proved in Pulido (2011). In addition, when futures contracts do overcome short selling constraints, we also investigate whether trading in only a single index of futures on the spot commodities (instead of all the futures on the spot commodities) is sufficient to complete the market. In general, the answer is again negative. However, hedging strategies involving all the futures on the spot commodities can be replaced by trading strategies using an index futures and only long trades on the futures contracts themselves. Here the long positions on the individual spot asset futures can be approximated by long positions on the spot commodities themselves, yielding an approximation to the desired result. The error term in this approximation depends on the magnitude of the market overpricing. This insight is particularly important for commodities that cannot be sold short and where futures on the individual asset are impractical, but where futures on an index of the commodities (a portfolio) can be traded. Two prime examples satisfying these conditions are: (i) residential homes and (ii) individual equities when short sale constraints are imposed.

The paper is organized as follows. In Section 2, we present two examples that motivate the discussion in the rest of the paper. In Section 3, we extend the Fundamental Theorem of Asset Pricing (FTAP) in markets with short sale prohibitions, as presented in Pulido (2011), to markets with positive interest rates and assets with cash flows. In Section 4, we study the implications 
that the FTAP imposes on futures prices. In Section 5, under various hypotheses, we characterize arbitrage-free futures prices when interest rates are identically zero. We show that futures prices exhibit more randomness than is present in the spot asset's price process, even under zero interest rates. We also give in Section 6 sufficient conditions under which futures contracts can be used to short the underlying asset, along with mathematical examples where this is not the case and where the spot asset cannot be hedged with traded futures. These examples complement those presented in Section 2. In Section 7, we extend the previous results to positive interest rate economies. Finally, in Section 8 we consider futures contracts on an index and show when trading an index futures is sufficient to overcome the short sale restrictions on the individual stocks themselves.

\section{PRELIMINARY EXAMPLES}

In this section we present two examples to show that under short sale restrictions, the common belief that the completeness property of the underlying asset price process (see Definition 6.1) is inherited by the futures price process is incorrect. Other examples will be presented in Section 6. These additional examples will better illustrate the results of Sections 5 and 6 (see Theorems 5.2 and 6.5). As we will see in Section 4, in a market with short sale prohibitions the set of risk-neutral measures for the futures market corresponds to the set of equivalent probability measures under which the discounted underlying asset price process (plus discounted cash flows) is a supermartingale and the futures price process is a local martingale. Furthermore, in the examples provided the futures price process is actually a martingale, which implies that futures prices do not have type 3 bubbles (see Jarrow et al. (2010)). For simplicity these examples also assume that the default free spot rate of interest is zero and the spot commodities have no cash flows.

The first example is in discrete time. It can be extended to a continuous time example with jumps as in Example 5.5.

Example 2.1 Suppose that for $t=1, \ldots, T$ the underlying asset price process is given by

$$
S_{t}=S_{0} \prod_{k=1}^{t}\left(1+R_{k}\right)
$$

where $S_{0}$ is a positive constant, under $P, R_{1}, \ldots, R_{T}$ are i.i.d random variables with $P\left(R_{1}=r\right)=$ $\frac{1}{2}, P\left(R_{1}=-r\right)=\frac{1}{2}$, and $0<r<1$. It is known that this financial market, where $S$ trades and the 
filtration considered is the minimal filtration generated by $S$, is complete under $P$. Fix $0<t^{*}<t$. Define a measure $Q^{*}$ such that for $n>t^{*}$, on the set $\left\{S_{t^{*}}=x\right\}$,

$$
Q^{*}\left(R_{n}=-r \mid \mathcal{F}_{n-1}\right)=p_{x} \in(1 / 2,1)
$$

and $Q^{*}\left(R_{n}=r \mid \mathcal{F}_{n-1}\right)=1-p_{x}$. Assume additionally that $Q^{*}$ coincides with $P$ over $\sigma\left(R_{1}, \ldots, R_{t^{*}}\right)$. $Q^{*}$ is a probability measure equivalent to $P$ such that $S$ is a $Q^{*}$-supermartingale. As we will prove in the next section, if we define a futures price process $F_{t, T}$ (see Definition 4.1) by the formula $F_{t, T}=E^{Q^{*}}\left[S_{T} \mid \mathcal{F}_{t}\right]$ (where $\mathcal{F}=\left(\mathcal{F}_{t}\right)_{t \geq 0}$ is the minimal filtration generated by $S$ ), the extended market where both $S$ and futures contracts on $S$ (with maturity $T$ ) trade, with short sale prohibitions on $S$, is arbitrage-free (see Proposition 4.2). Observe that in this example, on the set $\left\{S_{t^{*}}=x\right\}$

$$
\begin{aligned}
E^{Q^{*}}\left[S_{T} \mid \mathcal{F}_{t^{*}}\right] & =x \sum_{k=0}^{T-t^{*}}\left(\begin{array}{c}
T-t^{*} \\
k
\end{array}\right)(1-r)^{k}(1+r)^{T-t^{*}-k} p_{x}^{k}\left(1-p_{x}\right)^{T-t^{*}-k} \\
& =x\left((1-r) p_{x}+(1+r)\left(1-p_{x}\right)\right)^{T-t^{*}} \\
& =x\left((1+r)-2 r p_{x}\right)^{T-t^{*}} .
\end{aligned}
$$

Suppose that a fix positive constant c satisfies $0<c<S_{t^{*}}$ and

$$
r>1-\left(\frac{c}{S_{t^{*}}}\right)^{\frac{1}{T-t^{*}}}
$$

If we let

$$
p_{x}:=\frac{(1+r)-\left(\frac{c}{x}\right)^{\frac{1}{T-t^{*}}}}{2 r} \in(1 / 2,1),
$$

we have that $F_{t, T}=E^{Q^{*}}\left[S_{T} \mid \mathcal{F}_{t}\right]$ is identically equal to the constant $c$ for $t \leq t^{*}$ and the futures market is not complete under $Q^{*}$. Trivially, the futures cannot be used to hedge the underlying's price risk, either long or short. In other words, the futures market does not inherit the completeness property of the underlying market (see Figure 1). In other cases, for instance if the constants $p_{x}$ are all equal to a constant $p \in(1 / 2,1)$, the futures market is complete under $Q^{*}$.

For continuous processes we have the following example considered in Cox and Hobson (2005) when studying price bubbles. 
Example 2.2 (An Asset Price Process with a Bubble) Suppose that for $t<T$ the underlying asset price process $S$ is given by

$$
S=1+\mathcal{E}\left(\int_{0} \frac{d B_{s}}{\sqrt{T-s}}\right)
$$

where $B$ is a P-Brownian motion. We have that if

$$
X:=\int_{0}^{\cdot} \frac{d B_{s}}{\sqrt{T-s}}
$$

then

$$
[X]_{t}=\ln \left(\frac{T}{T-t}\right) \text { and } \lim _{t \rightarrow T}[X]_{t}=\infty, P \text {-a.s. }
$$

Since

$$
S_{t}=1+\exp \left(X_{t}-\frac{1}{2}[X]_{t}\right)=1+\exp \left([X]_{t}\left(\frac{X_{t}}{[X]_{t}}-\frac{1}{2}\right)\right)
$$

and $\lim _{t \rightarrow T} \frac{X_{t}}{[X]_{t}}=0$ (see Problem 2.9.3 and Theorem 3.4.6 in Karatzas and Shreve (1998a)), if we define $S_{T} \equiv 1$, then $S$ is a continuous strict local martingale on $[0, T]$, i.e. $S$ is a P-local martingale on $[0, T]$ that is not a P-martingale.

The market consisting of this risky asset price alone is complete. In this case $F_{t, T}:=E^{P}\left[S_{T} \mid \mathcal{F}_{t}\right]$ (where $\mathcal{F}=\left(\mathcal{F}_{t}\right)_{t}$ is the minimal filtration generated by $\left.S\right)$ is constant and equal to 1 . Again trivially, although the futures market is arbitrage-free, the futures cannot be used to hedge the underlying's price risk, and the futures market is not complete under $P$.

For markets with short sales restrictions, we would like to know when futures can be used to hedge the underlying asset's price risk. Before we answer this question in Section 6, we first investigate the dynamics of futures prices consistent with No Free Lunch with Vanishing Risk (NFLVR-S) when short sale prohibitions exist in the underlying market.

\section{THE FTAP WITH SHORT SALE PROHIBITIONS}

This section extends the results in Pulido (2011) to the case where the money market account's value is stochastic and risky assets have cash flows. We consider a finite trading horizon $[0, T]$ and assume that there are $N$ risky assets trading. We suppose, as in the seminal work of Delbaen and Schachermayer (1994), that the price processes of the $N$ risky assets are nonnegative locally 
bounded $P$-semimartingales on a stochastic basis $(\Omega, \mathcal{F}, \mathbb{F}, P)$, where $\mathbb{F}:=\left(\mathcal{F}_{t}\right)_{0 \leq t \leq T}$ satisfies the usual hypotheses. The probability measure $P$ denotes the statistical probability measure. We further assume that $\mathcal{F}_{0}$ is $P$-trivial and $\mathcal{F}_{T}=\mathcal{F}$. Hence, all random variables measurable with respect to $\mathcal{F}_{0}$ are $P$-almost surely constant and there is no additional source of randomness on the probability space. Given two probability measures $Q$ and $P$ on $\mathcal{F}$, we write $Q \sim P$ when $P$ and $Q$ are equivalent.

We denote by $S:=\left(S^{i}\right)_{1 \leq i \leq N}$ the $\mathbb{R}^{N}$-valued stochastic price processes for the risky assets. We assume that $S^{0}$, the value of a money market account, is a positive and predictable $P$-semimartingale bounded away from 0 . This is almost without loss of generality. In typical models, the money market account's value grows by continuously compounding at the default free spot rate of interest, i.e. the money market account's value equals a constant times the exponential of a Lebesque integral of the spot rate of interest across time. As such, the money market account's value is continuous a.e., even if the spot rate of interest follows a discontinuous sample path process. We denote by $\tilde{S}^{i}=\left(S^{0}\right)^{-1} S^{i}$ the discounted price process of asset $i$ for $1 \leq i \leq N$ and by $\tilde{S}:=\left(\tilde{S}^{1}, \ldots, \tilde{S}^{N}\right)$ the vector of discounted price processes.

We assume that for each asset $i, 1 \leq i \leq N$, there exists a cumulative cash flow process $D^{i}$, which is an $\mathbb{F}$-adapted $P$-semimartingale. We let $D:=\left(D^{1}, \ldots, D^{N}\right)$ be the vector of cumulative cash flow processes.

Also for $1 \leq i \leq N$ we define $\tilde{D}^{i}:=\frac{1}{S^{0}} \cdot D^{i}$ (throughout this paper the $\cdot$ symbol represents stochastic integration, see Protter (2005)). This integral is well defined since $S^{0}$ is nonnegative and bounded away from 0 . Finally, let $\tilde{D}:=\left(\tilde{D}^{1}, \ldots, \tilde{D}^{N}\right)$ be the vector of discounted cumulative cash flows.

Last, to capture short sale prohibitions on some of the traded assets, we assume that the first $d \leq N$ assets can be sold short in an admissible way (see Definition 3.1 below), but the last $N-d$ assets cannot, i.e. we define the admissible strategies as follows.

Definition 3.1 A vector valued process $H=\left(H^{1}, \ldots, H^{N}\right)$ is called an admissible trading strategy if

(i) $H \in L(\tilde{S}+\tilde{D})$, i.e. $H$ is integrable in the stochastic sense with respect to $\tilde{S}+\tilde{D}$ (see Protter (2005)).

(ii) $H_{0}=0$.

(iii) $(H \cdot(\tilde{S}+\tilde{D})) \geq-\alpha$ for some $\alpha>0$. 
(iv) $H^{i} \geq 0$ for all $i>d$.

We let $\mathcal{A}$ be the set of admissible trading strategies.

We define as in Pulido (2011) the sets

$$
\begin{gathered}
\mathcal{K}:=\left\{(H \cdot(\tilde{S}+\tilde{D}))_{T}: H \in \mathcal{A}\right\}, \\
\mathcal{C}:=\left(\mathcal{K}-L_{+}^{0}(P)\right) \cap L^{\infty}(P),
\end{gathered}
$$

where $L_{+}^{0}(P)$ is the space of equivalence classes of nonnegative finite random variables, and $L^{\infty}(P)$ is the space of $P$-essentially bounded random variables. With these sets, the arbitrage-free conditions of No Arbitrage under short sales prohibition (NA-S) and No Free Lunch with Vanishing Risk under short sales prohibition (NFLVR-S) are defined as follows. (NA-S) holds if $\mathcal{C} \cap L_{+}^{0}(P)=\{0\}$. (NFLVR-S) holds if $\overline{\mathcal{C}} \cap L_{+}^{0}(P)=\{0\}$, where $\overline{\mathcal{C}}$ is the closure of $\mathcal{C}$ with respect with the $\|\cdot\|_{\infty}$ norm in $L^{\infty}(P)$. Because these definitions are standard we provide no additional explanation. Throughout this paper whenever we write (NFLVR-S) we mean No Free Lunch with Vanishing Risk under short sales prohibition.

The following theorem is an immediate consequence of Theorem 3.10 in Pulido (2011) and the fact that $S^{0}$ is a positive semimartingale bounded away from 0 .

Theorem 3.2 Under the additional assumption that for all $i, \tilde{D}^{i}$ is a locally bounded semimartingale under $P$, the condition of $(N F L V R-S)$ is equivalent to

$$
\mathcal{M}_{\text {sup }}(\tilde{S}+\tilde{D}) \neq \emptyset
$$

where $\mathcal{M}_{\text {sup }}(\tilde{S}+\tilde{D})$ is the set of probability measures $Q \sim P$ such that $\tilde{S}^{i}+\tilde{D}^{i}$ is a $Q$-local martingale for $1 \leq i \leq d$ and $\tilde{S}^{i}+\tilde{D}^{i}$ is a $Q$-local supermartingale for $d<i \leq N$. If in addition $\tilde{D}^{i}$ is bounded from below, then $\tilde{S}^{i}+\tilde{D}^{i}$ is a $Q$-supermartingale for $Q$ in $\mathcal{M}_{\text {sup }}(\tilde{S}+\tilde{D})$.

Proof. This corresponds to Theorem 3.10 in Pulido (2011) after replacing $S$ by $\tilde{S}+\tilde{D}$. The only difference is that $\tilde{S}+\tilde{D}$ is not always nonnegative. However, a careful inspection of the proof of Proposition 3.2 in Pulido (2011) shows that the conclusion of this theorem holds.

Although the previous theorem seems to be folklore knowledge (see for instance Cvitanić and Karatzas (1993), Jouini and Kallal (1995), Schürger (1996), Frittelli (1997), Pham and Touzi 
(1999), Napp (2003)), we could not find a proof at this level of generality for semimartingales with jumps. Our proof relies on a modification of a known proposition by Ansel and Stricker (see Proposition 3.3 in Ansel and Stricker (1994)), which is proved in Pulido (2011). This theorem will be used to study the conditions under which futures contracts can be used to overcome short sale prohibitions on the underlying commodities.

\section{ARBITRAGE-FREE FUTURES PRICES}

This section explores the implications that the Fundamental Theorem of Asset Pricing (FTAP) under short sale prohibitions (Theorem 3.2) has on futures prices and their hedging strategies. Initially, to simplify our notation, we assume that there is only one risky asset trading and that this asset cannot be sold short (i.e. we assume that $d=0, N=1$ ). We denote by $S$ the price of the risky asset and call it the underlying asset price process or spot price process. We further assume that there are no cash flows associated to $S$. As we did in the previous section, we denote by $S^{0}$ the price of the money market account, and assume that it is a positive predictable $P$ semimartingale bounded away from zero. $\tilde{S}:=\left(S^{0}\right)^{-1} S$ therefore corresponds to the discounted price process of the risky asset.

The previous section shows that (NFLVR-S) guarantees the existence of a probability measure in $\mathcal{M}_{\text {sup }}(\tilde{S})$ (see Theorem 3.2). This set of probability measures contains the set of measures $\mathcal{M}_{\text {loc }}(\tilde{S})$ defined by

$$
\mathcal{M}_{\text {loc }}(\tilde{S}):=\{Q \sim P: \tilde{S} \text { is a } Q \text {-local martingale }\}
$$

Furthermore, the Fundamental Theorem of Asset Pricing (FTAP) presented in Delbaen and Schachermayer (1994), shows that this later set $\mathcal{M}_{l o c}(\tilde{S}) \neq \emptyset$ if and only if the condition of (NFLVR-S) holds for admissible trading strategies, without restriction (iv) in Definition 3.1. Hence, with the short sale prohibitions on $S$, the set of risk neutral measures is enlarged from $\mathcal{M}_{\text {loc }}(\tilde{S})$ to $\mathcal{M}_{\text {sup }}(\tilde{S})$. This makes intuitive sense. If the set of trading strategies is restricted by short sale constraints, then the set of price processes consistent with no-arbitrage should increase, and it does.

Suppose that for some $Q^{*} \in \mathcal{M}_{\text {sup }}(\tilde{S}), \tilde{S}_{t}^{*}:=E^{Q^{*}}\left[\tilde{S}_{T} \mid \mathcal{F}_{t}\right]$ corresponds to the discounted fundamental price of $S$ at time $t$. It has been argued that in markets with short sale prohibitions, under certain hypotheses on agents' beliefs, the measure $Q^{*}$ is a strict supermartingale measure, 
in the sense that $\tilde{S}_{0}>\tilde{S}_{0}^{*}$. This is known as the overpricing hypothesis (see for instance Miller (1977), Chapter 7 of Fabozzi (2004), Boulton and Braga Alves (2010) and Brown and Rogers (2012)). In the case when $Q^{*}$ is a strict local martingale measure, the asset $S$ has a price bubble (see Jarrow et al. (2007), Jarrow et al. (2010)). This case has been studied extensively (see for instance Delbaen and Schachermayer (1995), Cox and Hobson (2005), Madan and Yor. (2006), Jarrow et al. (2007), Ekström and Tysk (2009), Jarrow et al. (2010), Pal and Protter (2010), Mijatović and Urusov (2012), Ruf (2012)).

For hedging, it is often argued that by trading in futures markets, short sale prohibitions can be overcome (see Chapter 3 of Fabozzi (2004) and Chapter 7 of Duffie (1989)). The previous examples (see Section 2) show that this is not always true. In the subsequent sections we study both the consequences of short sale prohibitions and the overpricing hypothesis on the ability of hedging strategies using futures to generate a short position in the underlying asset price process $S$. Our analysis differs from that used in the bubbles literature because we consider supermartingale measures, rather than local martingale measures. Consequently, our conclusions and examples apply to a larger variety of models, including both the Black-Scholes and discrete time models.

We define futures contracts as in Karatzas and Shreve (1998b) and Jarrow and Protter (2009).

Definition 4.1 A futures contract on a risky asset with price process $S$ and maturity time $T$ is a financial instrument with cash flows $d F_{t, T}$ such that

(i) $F_{t, T}$ is a nonnegative $\mathbb{F}$-adapted $P$-semimartingale with $F_{T, T}=S_{T}$, and

(ii) the market price of the cash flows $\left(d F_{t, T}\right)_{t}$ is zero at all times.

$F_{t, T}$ is called the futures price process.

Condition (ii) in this definition makes the futures price process dependent on the market price. Note that the futures price process is different from the market price of the futures contract which is always zero. Using the notation of Section 3, if we denote by $S^{2}$ a futures contract on $S$ with maturity $T$, the discounted market price of $S^{2}$ is equal to 0 (i.e. $\tilde{S}^{2}=0$ ) and the cash flow associated with $S^{2}$ is $d F_{, T}$ (i.e. $d D^{2}=d F_{\cdot, T}$ ). Investors are allowed to take both long and short positions in a futures contract.

Intuitively a long futures contract on $S$ at time $t$ obligates the holder to purchase the risky asset at time $T$ at a fixed price $F_{t, T}$, specified at time $t$. The payment is arranged in different 
installments determined by the fluctuations of the futures prices over the contract's life. In practice this is accomplished by using a margin account. When futures prices increase, the increment is deposited in the margin account. And when futures prices decrease, the negative increment is withdrawn from the margin account. The futures margin account is said to be marked-to-market. The cumulative changes in the futures price between time $t$ and the contract's maturity sum to the spot price of the asset at time $T$ less the original fixed price $F_{t, T}$.

Unlike shorting the underlying asset, taking a short position in a futures contract is unrestricted and it is equivalent to selling the futures contract. A short position in a futures contract is entitled to a stream of cash flows exactly opposite to a long position. In practice, a futures transaction is made through a clearing house where for each seller the clearing house finds a buyer on the other side of the contract. Acting as a middle man between the buyer and seller, the clearing house reduces counterparty risk, and it also facilitates reduced transaction costs and increased market liquidity.

Finally, there is no cash flow paid when entering a long or short position in a futures contract (see (ii) in Definition 4.1), other than the necessity of setting up a margin account (for a detailed exposition we refer the reader to Duffie (1989)). However, under our assumptions such margin requirements impose no additional restrictions on the set of trading strategies. This is because a margin account is equivalent to holding the money market account, and borrowing and lending is unrestricted in our model.

\subsection{NFLVR-S IN THE EXTENDED MARKET}

This section presents some necessary and sufficient conditions on futures price processes under which the underlying asset price process $S$ and the futures contract on $S$ with maturity $T$ satisfy the no arbitrage condition of (NFLVR-S).

Proposition 4.2 If the futures price process $F_{t, T}$ is a $Q$-local martingale for some $Q \in \mathcal{M}_{\text {sup }}(\tilde{S})$ then the extended market where both the underlying risky asset $S$ and the futures contract on $S$ trade satisfies (NFLVR-S). Conversely, if furthermore $S^{0}$ is locally bounded from above, $\tilde{D}:=$ $\left(S^{0}\right)^{-1} \cdot F_{\cdot, T}$ is locally bounded, and the extended market satisfies (NFLVR-S), then there exists a $Q \in \mathcal{M}_{\text {sup }}(\tilde{S})$ such that $F_{t, T}$ is a $Q$-local martingale.

Proof. $(\Rightarrow)$ We have that $\tilde{D}=\left(S^{0}\right)^{-1} \cdot F_{\cdot, T}$ is a $Q$-local martingale since $\left(F_{t, T}\right)_{t}$ is a $Q$-local martingale and $\left(S^{0}\right)^{-1}$ is bounded $\left(S^{0}\right.$ is bounded away from 0$)$. The conclusion follows from 
Theorem 3.2.

$(\Leftarrow)$ Assume that $S^{0}$ is locally bounded from above and $\tilde{D}=\left(S^{0}\right)^{-1} \cdot F_{\cdot, T}$ is locally bounded. By Theorem 3.2 there exists $Q \in \mathcal{M}_{\text {sup }}(\tilde{S})$ such that $\tilde{D}$ is a $Q$-local martingale, and since $F_{t, T}=$ $F_{0, T}+\left(S^{0} \cdot \tilde{D}\right)_{t}$ and $S^{0}$ is locally bounded, $F_{\cdot, T}$ is a $Q$-local martingale as well.

Remark 4.3 In the above proof the process $\tilde{D}$ corresponds to the discounted cash flows from a futures contract on $S$ with maturity $T$. Notice that in order for the extended market to satisfy $(N F L V R-S)$ it suffices to ensure the existence of a measure $Q \in \mathcal{M}_{\text {sup }}(\tilde{S})$ such that $\left(F_{t, T}\right)_{t}$ is a $Q$ local martingale and not necessarily a Q-martingale (see Jarrow and Protter (2009)). Also, since usually $\left|\mathcal{M}_{\text {sup }}(\tilde{S})\right|>1$ this proposition shows that the futures price process is not uniquely determined by the underlying asset price process and (NFLVR-S). Indeed, for arbitrary $Q \in \mathcal{M}_{\text {sup }}(\tilde{S})$

$$
F_{t, T}:=E^{Q}\left[S_{T} \mid \mathcal{F}_{t}\right]
$$

defines the futures price of a futures contract in such a way that the extended market satisfies (NFLVR-S). In principle, these futures price processes could differ across different risk neutral measures (see Example 4.5 below).

Taking into account the remarks just made we impose the following assumption on the futures contract on $S$.

\section{Assumption 4.4}

The futures price process $F_{\cdot, T}$ is a $Q^{*}$-martingale, for at least one $Q^{*} \in \mathcal{M}_{\text {sup }}(\tilde{S})$.

This implies that the extended market including both $S$ and $F$ satisfies (NFLVR-S) and the futures contract on $S$ does not have a bubble with respect to $Q^{*} \in \mathcal{M}_{\text {sup }}(\tilde{S})$ (see Jarrow et al. (2007), Jarrow et al. (2010)).

The purpose of the next example is to illustrate, under Assumption 4.4, the relationship between the arbitrage-free futures price and the underlying asset's price processes. This example shows that the futures price process inherits the dynamics of the underlying asset price process if additional assumptions are made on the measure $Q^{*}$ of Assumption 4.4 (see (4.2) and (4.3) below). Furthermore, under these additional assumptions, an explicit formula characterizing the 
difference between the underlying asset price process and the futures price can be derived (see Equation (4.4) below). The example motivates Theorem 5.2. It is important to point out that Assumption 4.4 is not a necessary condition for the futures price process to inherit the structure of the asset's price process. For instance, in Example 2.2, $F_{\cdot, T}=S$ defines a futures price process consistent with (NFLVR-S) which has the same dynamics as the spot price, but Assumption 4.4 does not hold in this case.

Example 4.5 (The Black-Scholes Model with Short Sale Prohibitions) Assume that

$$
d S_{t}=S_{t}\left(\mu d t+\sigma d B_{t}\right)
$$

where $B_{t}$ is an $\mathbb{F}$-Brownian Motion under $P$, and $\mu$ and $\sigma$ are positive constants, i.e.

$$
S_{t}=S_{0} \exp \left(\sigma B_{t}+\left(\mu-\frac{1}{2} \sigma^{2}\right) t\right) .
$$

Assume that the default free spot rate of interest is identically equal to 0 so that $\tilde{S}=S$. Furthermore, suppose that $\mathbb{F}$ is the filtration generated by $B$, and that $Q^{*} \in \mathcal{M}_{\text {sup }}(\tilde{S})$. By the martingale representation theorem for Brownian Motion (see Theorem IV-43 in Protter (2005)) we have that there exists a predictable process $\eta$ such that for all $t \in[0, T]$,

$$
Z_{t}:=E^{P}\left[\frac{d Q^{*}}{d P} \mid \mathcal{F}_{t}\right]=\mathcal{E}(\eta \cdot B)_{t}
$$

Girsanov's theorem (Theorem III-40 in Protter (2005)) implies that under $Q^{*}$, S has the semimartingale decomposition

$$
\begin{aligned}
d S_{t} & =\sigma S_{t}\left(d B_{t}-\eta_{t} d t\right)+S_{t}\left(\sigma \eta_{t}+\mu\right) d t \\
& =\sigma S_{t} d B_{t}^{*}+S_{t}\left(\sigma \eta_{t}+\mu\right) d t
\end{aligned}
$$

where $B^{*}$ is an $\mathbb{F}$-Brownian Motion under $Q^{*}$. Since $Q^{*} \in \mathcal{M}_{\text {sup }}(\tilde{S})$, we have that $S$ is a $Q^{*}$ supermartingale, and we conclude that the finite variation process

$$
\int_{0}^{\cdot} S_{s}\left(\sigma \eta_{s}+\mu\right) d s
$$

is indistinguishable from a nonincreasing process and $\eta \leq-\frac{\mu}{\sigma} P \otimes \lambda$-almost surely, where $\lambda$ denotes 
Lebesgue measure on $[0, T]$. Recall that $\frac{\mu}{\sigma}$ is commonly known as the market price of risk.

Now, according to Assumption 4.4 the futures price process of a futures contract on $S$ with maturity $T$ is given by

$$
\begin{aligned}
F_{t, T} & =E^{Q^{*}}\left[S_{T} \mid \mathcal{F}_{t}\right] \\
& =E^{Q^{*}}\left[S_{0} \exp \left(\sigma B_{T}^{*}+\int_{0}^{T}\left(\sigma \eta_{s}+\mu-\frac{\sigma^{2}}{2}\right) d s\right) \mid \mathcal{F}_{t}\right] .
\end{aligned}
$$

In order to obtain a more explicit formula for the futures price process at time $t$ we could impose the additional assumption that

$$
\int_{t}^{T} \sigma \eta_{s} d s \text { is } \mathcal{F}_{t} \text {-measurable. }
$$

In essence, this additional assumption implies that the futures price process has no additional randomness over the contract's entire maturity, other than that present in the price process itself. In this case we can conclude that

$$
F_{t, T}=S_{0} \exp \left(\int_{0}^{T}\left(\sigma \eta_{s}+\mu\right) d s\right) \mathcal{E}\left(\sigma B^{*}\right)_{t} .
$$

Under no short sale prohibitions and zero interest rates with $\eta \equiv-\frac{\mu}{\sigma}$, the dynamics of the futures price process is given by

$$
S_{0} \mathcal{E}\left(\sigma B^{*}\right)_{t}
$$

Hence, in a market with short sale prohibitions, if the overpricing hypothesis and (4.2) hold, then at time $t$ the futures price process has the additional discounting factor

$$
\exp \left(\int_{0}^{T}\left(\sigma \eta_{s}+\mu\right) d s\right)
$$

It is interesting to note that in this case

$$
\begin{aligned}
\frac{F_{t, T}}{S_{t}} & =\frac{\exp \left(\int_{0}^{T}\left(\sigma \eta_{s}+\mu\right) d s\right) \mathcal{E}\left(\sigma B^{*}\right)_{t}}{\exp (\mu t) \mathcal{E}(\sigma B)_{t}} \\
& =\exp \left(\int_{t}^{T}\left(\sigma \eta_{s}+\mu\right) d s\right)
\end{aligned}
$$


Since $F_{\cdot, T}$ and $S$ are observable, one can estimate $\eta+\frac{\mu}{\sigma}$ from market price observations. Of course, in order for (4.4) to hold, we are not taking into consideration interest rates (see Section 7 below) and we are adding an additional assumption on the process $\eta$, namely (4.2) (see Theorem 5.2 below). Equation (4.4) agrees with the empirical evidence on the effect of short sales restrictions on futures prices and stock returns (see for instance Fung and Draper (1999) and Boulton and Braga Alves (2010)).

Remark 4.6 Condition (4.2) is a strong assumption. It effectively assumes that the futures price process exhibits no additional randomness over the contract's entire maturity, other than that reflected in the underlying asset's price process itself. There is no reason to believe this must be true a priori. Equivalently, condition (4.2) essentially assumes that the future evolution of the process $\sigma \eta_{u}$ for $t \leq u \leq T$ is observable at time $t$. Again, under the filtration generated by the Brownian filtration, there is no reason to believe that this must be true a priori.

This condition might hold if one enlarges the original filtration to include knowledge of $\left(\eta_{u}\right)_{t \leq u \leq T}$ by appealing to the theory of enlargement of filtrations. A simple way to do this would be to include the entire process $\left(\eta_{u}\right)_{0 \leq u \leq T}$ in $\mathcal{F}_{0}$ via what is known as an initial enlargement, using Jacod's Criterion (see for example Chapter VI of Protter (2005)). But this approach seems inappropriate for processes such as $\eta$ which arise through martingale representation. An alternative solution might be to include gradually the process $\eta$ in the filtration via a dynamic enlargement, but this theory is still in its infancy, see Kchia and Protter (2011).

\section{ZERO-INTEREST RATES}

This section generalizes Example 4.5 and characterizes the relation between the futures and asset price processes. As in the previous section, we continue to assume that the spot rate of interest is identically equal to 0 so $S=\tilde{S}$. We can describe the dynamics of the futures prices under Assumption 4.4. Before we do so let's recall the following lemma.

Lemma 5.1 Suppose that the supermartingale $S>0$. Then $S$ has one and only one multiplicative decomposition of the form $S=L D$ where $L$ is a positive $Q^{*}$-local martingale and $D$ is a positive, predictable and nonincreasing process with $D_{0}=S_{0}$. Furthermore, if $S=S_{0}+N+V$ is the Doob-Meyer decomposition of the $Q^{*}$-supermartingale $S$, with $N$ a $Q^{*}$-local martingale, and $V$ a 
predictable and nonincreasing process with $N_{0}=V_{0}=0$ then

$$
\begin{gathered}
L=\mathcal{E}\left(\left(\frac{1}{S_{-}+\Delta V}\right) \cdot N\right) \\
D=S_{0}\left(\mathcal{E}\left(-\left(\frac{1}{S_{-}+\Delta V}\right) \cdot V\right)\right)^{-1} .
\end{gathered}
$$

Proof. The proof of this result can be found in Section VI-2-a of Jacod (1979). See also Section II-8b of Jacod and Shiryaev (2003).

By using this lemma and the ideas contained in Example 4.5 we have the following result.

Theorem 5.2 Suppose that $S>0$ and Assumption 4.4 holds. With the notation of Lemma 5.1 we have the following.

1. If $N$ is a $Q^{*}$-martingale then $F_{t, T}=S_{0}+N_{t}+E^{Q^{*}}\left[V_{T} \mid \mathcal{F}_{t}\right]$. Furthermore, if $V_{T} \in \mathcal{F}_{t_{0}}$ for some $t_{0}<T$, then $F_{t, T}=S_{0}+N_{t}+V_{T}$ for all $t \in\left[t_{0}, T\right]$.

2. If $L$ is a $Q^{*}$-martingale and $D_{T} \in \mathcal{F}_{t_{0}}$ for some $t_{0}<T$, then $F_{t, T}=L_{t} D_{T}$ for all $t \in\left[t_{0}, T\right]$.

Proof. This theorem follows simply from the expression $F_{t, T}=E^{Q^{*}}\left[S_{T} \mid \mathcal{F}_{t}\right]$.

Remark 5.3 Since this result does not take into consideration interest rates, the futures price coincides with the forward price. Recall that the forward price at time $t$ is the value of $K$ such that the price of a forward contract with delivery price $K$ and maturity $T$ has market price 0 at time $t$. Assuming that there is no bubble for the forward contract with respect to $Q^{*}$ this holds if and only if $E^{Q^{*}}\left[S_{T}-K \mid \mathcal{F}_{t}\right]=0$ and $K=E^{Q^{*}}\left[S_{T} \mid \mathcal{F}_{t}\right]$.

Remark 5.4 (Black-Scholes continued) If we assume that the hypotheses of the previous theorem hold, then in the previous Black-Scholes example, the futures price process differs from the asset price process for $t \in\left[t_{0}, T\right]$ by the factor

$$
\frac{F_{t, T}}{S_{t}}=\frac{D_{T}}{D_{t}}
$$

When using futures contracts to hedge the asset price process under short sale prohibitions it is important to recognize the existence of this additional factor. Observe that in Example 4.5, $D_{t}=\exp \left(\int_{0}^{t}\left(\sigma \eta_{s}+\mu\right) d s\right)$ and the previous theorem agrees with (4.3). 
Although the conclusion of the previous theorem might seem trivial at first, Theorem 5.2 motivates the discussion in the next section. As the following example shows, the dynamics of the futures price process on the interval $\left[0, t_{0}\right)$ might be very different from the underlying asset's price process and consequently not inherit properties such as completeness (see Definition 6.1 below) on this interval.

Example 5.5 Suppose that $\Omega=\left(\omega_{1}, \omega_{2}, \omega_{3}, \omega_{4}\right)$ and $T=3$. Suppose that the price process of an asset that cannot be sold short is given by $S$, where $S_{t} \equiv \frac{21}{16}$ for $0 \leq t<1 ; S_{t}\left(\omega_{1}\right)=S_{t}\left(\omega_{2}\right)=\frac{11}{8}$, $S_{t}\left(\omega_{3}\right)=S_{t}\left(\omega_{4}\right)=\frac{10}{8}$ for $1 \leq t<2 ; S_{t}\left(\omega_{1}\right)=\frac{7}{4}, S_{t}\left(\omega_{2}\right)=\frac{1}{4}, S_{t}\left(\omega_{3}\right)=\frac{3}{2}, S_{t}\left(\omega_{4}\right)=\frac{1}{2}$ for $2 \leq t \leq 3$. Let $\mathbb{F}$ be the minimal filtration generated by $S$.

We have in this case that $P$ given by $P\left(\omega_{1}\right)=P\left(\omega_{3}\right)=\frac{3}{8}, P\left(\omega_{2}\right)=P\left(\omega_{4}\right)=\frac{1}{8}$ is a martingalemeasure for $S$. Furthermore, $P$ is the only measure that makes $S$ a martingale.

Next, suppose that $Q^{*}$ is given by $Q^{*}\left(\omega_{1}\right)=Q^{*}\left(\omega_{2}\right)=Q^{*}\left(\omega_{3}\right)=Q^{*}\left(\omega_{4}\right)=\frac{1}{4}$. Then $Q^{*} \in$ $\mathcal{M}_{\text {sup }}(S)$. We have that $E^{Q^{*}}\left[S_{t} \mid \mathcal{F}_{1}\right] \equiv 1$ for $2 \leq t \leq 3$. It is easy to see that the canonical decomposition of the special semimartingale $S$ relative to $Q^{*}$ is $S=S_{0}+N+V$, where $V_{t} \equiv 0$ for $0 \leq t<2$ and $V_{t}=1-S_{1}$ for $2 \leq t \leq 3, N_{t}=0$ for $0 \leq t<1, N_{t}=S_{1}-S_{0}$ for $1 \leq t<2$ and $N_{t}=S_{2}+\left(S_{1}-1\right)-S_{0}$ for $2 \leq t \leq 3$. We have that

$$
\begin{gathered}
\left(\frac{1}{S_{-}+\Delta V} \cdot N\right)_{t}=\frac{S_{1}-S_{0}}{S_{0}+0} 1_{[1,3]}(t)+\frac{S_{2}-1}{S_{1}+\left(1-S_{1}\right)} 1_{[2,3]}(t) \\
=\frac{S_{1}-S_{0}}{S_{0}} 1_{[1,3]}(t)+\left(S_{2}-1\right) 1_{[2,3]}(t), \\
\left(\frac{1}{S_{-}+\Delta V} \cdot V\right)_{t}=\frac{1-S_{1}}{S_{1}+\left(1-S_{1}\right)} 1_{[2,3]}(t) \\
=\left(1-S_{1}\right) 1_{[2,3]}(t) .
\end{gathered}
$$

The conditions of Theorem 5.2 are satisfied in this case with $t_{0}=1$.

Observe that in this case $F_{t, T} \equiv 1$ for $0 \leq t<2$. The multiplicative decomposition of $S$ (see Lemma 5.1) is given by

$$
D= \begin{cases}S_{0} & \text { if } 0 \leq t<2 \\ \frac{S_{0}}{S_{1}} & \text { for } 2 \leq t \leq 3\end{cases}
$$




$$
L= \begin{cases}1 & \text { for } 0 \leq t<1 \\ \frac{S_{1}}{S_{0}} & \text { for } 1 \leq t<2 \\ \frac{S_{1} S_{2}}{S_{0}} & \text { for } 2 \leq t \leq 3\end{cases}
$$

and the conclusion of the theorem does not hold for $t \in[0,1)$. A discrete time representation of this example can be found in Figure 2.

In this example the futures contract cannot be used to hedge positions on $S$ before time 2 . In the next section we prove that when the hypothesis of Theorem 5.2 holds with $t_{0}=0$, market completeness in the underlying asset price process is inherited by the futures market. We will also provide a striking continuous time example (Example 6.9) when this fails, which complements the examples provided in Section 2 and Example 5.5 above.

\section{THE REPRESENTATION PROPERTY}

Suppose that the financial market is complete without short sale prohibitions. It is commonly believed that, without taking into account interest rates, the introduction of trading in futures contracts completes the market. In this section we provide sufficient conditions under which this belief is true, and we provide counterexamples to show that it is not true in general.

The concept of a complete market is usually defined in terms of a predictable representation property for the assets' price processes (see for instance the seminal works of Harrison and Pliska (1981) and Harrison and Pliska (1983)). In this section we discuss some conditions under which this property on the underlying asset price process is inherited by the futures price process. To keep the notation simple, as before we assume that there is only one underlying asset price process $S$ trading in the market and that the spot interest rate and associated cash flows are identically zero. We furthermore assume that there exists a $P^{*} \in \mathcal{M}_{l o c}(S)$. In other words, we assume that $S$ satisfies (NFLVR-S) for admissible integrands without short sale prohibitions.

Definition 6.1 (Market Completeness) We say that the financial model is complete under $P^{*}$, or that $S$ has the completeness property with respect to $P^{*}$, if every $P^{*}$-local martingale $M$ can be expressed as $M=M_{0}+(H \cdot S)$ for some $H \in L(S)$.

Given the results in the previous section it is interesting to study whether the local martingale part $N$, of the Doob-Meyer decomposition of $S$ under $Q^{*}$, inherits the completeness property of 
the process $S$. This, in turn, will enable us to derive sufficient conditions under which the futures market inherits the completeness property from the underlying asset market (see Theorem 6.5 below). In order to prove this result in general, we first have to prove it for continuous processes.

Theorem 6.2 Assume that $S$ is a continuous process that has the completeness property with respect to $P^{*}$, and $N$ is the local martingale part in the canonical decomposition of $S$ under $Q^{*}$. Then $N$ has the completeness property with respect to $Q^{*}$.

Proof. The proof of this result when $S$ is a $P^{*}$-Brownian motion can be found in Proposition 5.8.1.2. of Chesney et al. (2009). The proof can be mimicked step by step for any continuous process and the conclusion follows from Girsanov's Theorem (Theorem III-40 in Protter (2005)).

The following result generalizes the previous theorem. It shows that in general the local martingale part of the canonical decomposition of $S$ under $Q^{*}$ inherits the completeness property of the underlying asset price process $S$.

Theorem 6.3 Assume that $S$ has the completeness property with respect to $P^{*}$, and $N$ is the local martingale part in the canonical decomposition of $S$ under $Q^{*}$. Then $N$ has the completeness property with respect to $Q^{*}$.

Proof. We let $(0, c, K, A)$ be a good version of the semimartingale characteristics as in (A.1) of Appendix A, with $P$ replaced by $P^{*}$. By localization, it is enough to show that any $Q^{*}$-martingale $X$ in $\mathcal{H}_{1}\left(Q^{*}\right)$ (see Protter (2005) p.195) with $X_{0}=0$ can be written as $X=H \cdot N$ for some $H \in L(N)$. For a semimartingale $Z$ with good version of the semimartingale characteristics $\left(b^{Z}, c^{Z}, K^{Z}, A^{Z}\right)$ we denote by $L_{c, Z}(\omega, t)$ the eigenspace of $c^{Z}(\omega, t)$ corresponding to the eigenvalue 1 and by $L_{d, Z}(\omega, t)$ the support of the kernel $K^{Z}(\omega, t)$. By Theorem 4.82 in Jacod (1979), to show the above mentioned representation for $N$ it is enough to show that:

(i) Any continuous $Q^{*}$-martingale $Y$ in $\mathcal{H}_{1}\left(Q^{*}\right)$ can be written as $K \cdot N^{c}$ for some predictable process $K$, where $N^{c}$ is the continuous $Q^{*}$-local martingale part of $N$.

(ii) $L_{c, N}(\omega, t) \cap L_{d, N}(\omega, t)=\{0\} M_{A^{N}}^{Q^{*}}$ a.s. in $(\omega, t)$ (see Jacod $\left.(1979)\right)$.

By the same theorem, we have the same properties for $S$ with respect to $P^{*}$. (i) follows by Theorem 6.2 and the fact that $N^{c}$ is the $Q^{*}$-local martingale part of $S^{c}$. To see (ii), observe that 
$L_{c, N}$ coincides with $L_{c, S}$, that $L_{d, N}$ is a subset of $L_{d, S}$ by equation (B.1) of Appendix B, and that we can take $A:=A^{S}=A^{N}$. Hence, $L_{c, N}(\omega, t) \cap L_{d, N}(\omega, t)=\{0\} M_{A}^{P^{*}}$-a.s. in $(\omega, t)$. Since $Q^{*} \sim P^{*}$, this implies that $L_{c, N}(\omega, t) \cap L_{d, N}(\omega, t)=\{0\} M_{A}^{Q^{*}}-$ a.s. in $(\omega, t)$.

Remark 6.4 This theorem shows that the completeness property of the local martingale part in the $Q^{*}$-canonical decomposition of $S$ is inherited from the completeness property of $S$ with respect to $P^{*}$. Theorem $11.29 \mathrm{in} \mathrm{Jacod} \mathrm{(1979)} \mathrm{shows} \mathrm{that} \mathrm{this} \mathrm{condition} \mathrm{is} \mathrm{necessary} \mathrm{for} \mathrm{the} \mathrm{supermartingale}$ measure $Q^{*}$ to be an extreme point among all the measures in $\mathcal{M}_{\text {sup }}(S)$, but in general the two conditions are not equivalent. Hence, even for measures that are not extreme in $\mathcal{M}_{\text {sup }}(S)$, the completeness property of the local martingale is inherited from the underlying process $S$.

Theorem 6.3 allows us to provide a set of sufficient conditions that guarantees the futures market is complete, even when asset price bubbles exist.

Theorem 6.5 Assume the hypotheses of Theorem 5.2 (2) hold with $t_{0}=0$ and that the financial market where $S$ trades is complete under $P^{*}$. Then, the futures market where a futures contract with price process $F_{t, T}=E^{Q^{*}}\left[S_{T} \mid \mathcal{F}_{t}\right]$ trades is complete under $Q^{*}$, i.e. all $Q^{*}$-local martingales are of the form $x+\left(H \cdot F_{\cdot, T}\right)$ for some constant $x$ and $H \in L\left(F_{\cdot, T}\right)$.

Proof. If the hypotheses of Theorem $5.2(2)$ hold with $t_{0}=0$ then the futures price process' dynamics are given by

$$
d F_{t, T}=S_{0} D_{T} d L_{t}=\frac{S_{0} D_{T} L_{t-}}{S_{t-}+(\Delta V)_{t}} d N_{t}
$$

where $S=S_{0}+N+V$ is the Doob-Meyer decomposition of the $Q^{*}$-supermartingale $S$. It follows easily from equation (6.1) and Theorem 6.3 that in this case the futures market is complete with respect to $Q^{*}$.

Theorem 6.5 states that if the discount factor $D_{T}$ is $\mathcal{F}_{0}$ measurable, the interest rates are zero, the spot price process is strictly positive, and the futures price process can be written as a conditional expectation, then trading in the futures price process completes the market when the underlying spot price process has restricted short sales. This theorem is perhaps the source of the common belief mentioned earlier because in the classical literature on futures contracts, these three assumptions are common. Indeed, stochastic interest rates were usually not considered so no distinction was usually made between forward and futures contracts, and futures prices were defined as the conditional expectation in Assumption 4.4. 
When we relax the hypotheses of this theorem, the completeness property of the underlying asset price process need not be inherited by the futures price process. As we showed in the examples of Section 2, the common belief that trading in futures completes the market when short selling in the spot asset is prohibited is false in general.

Example 6.6 In Example 2.1 the conclusion of Theorem 6.5 does not apply. In this case the discount factor $D_{T}$ is not $\mathcal{F}_{0}$-measurable.

Example 6.7 It is easy to check that the model presented in Example 5.5 is complete under $P=P^{*}$. However, since $F_{\cdot, T}$ is constant on the interval $[0,2)$ the futures market is not complete under $Q^{*}$. As we already pointed out, in this example the hypothesis of Theorem 5.2 does not hold with $t_{0}=0$, because the discount factor $D_{T}$ is not $\mathcal{F}_{0}$-measurable. This example complements Example 2.1, because in this case the probability measure $Q^{*}$ at time 1 is independent of the level of the underlying asset price, while in Example 2.1 the probability measure $Q^{*}$ depends on the level of the underlying asset price at time $t^{*}$.

Example 6.8 In Example 2.2 the conclusion of Theorem 6.5 fails. In this case even though the discount factor $D$ is constant and is identically equal to $S_{0}$, the local martingale factor $L\left(=S / S_{0}\right)$ is not a martingale with respect to the measure $Q^{*}(=P)$.

The following example is even more striking, and it is motivated by Example 2.1. It shows that the futures price process can lack the completeness property even if the underlying asset price process is a continuous martingale under $P^{*}$ satisfying the completeness property with respect to its minimal filtration. This example is the generalization to continuous time of the idea behind Example 2.1, where after a certain time the measure $Q^{*}$ is dependent on the level of the underlying asset price process at a fixed time $t^{*}$.

Example 6.9 In this example, we first construct a price process for a risky asset on a complete and filtered probability space $\left(\Omega, \mathcal{G}, \mathbb{G}, P^{*}\right)$. We denote this price process as $S$ and we show it has the martingale representation property with respect to its own filtration $\mathbb{F}$. We then construct a futures process $F$ for $S$ and show that, under $\mathbb{F}$, the futures process does not have martingale representation. This shows that martingale representation (and hence market completeness) is not - in general - inherited by a futures process, even if the risky asset price process has the property.

$W e$ begin with the construction of $S$, which is slightly complicated. We let $W$ be a standard Brownian motion on $\left(\Omega, \mathcal{G}, \mathbb{G}:=\left(\mathcal{G}_{t}\right), P^{*}\right)$. Given $x \in \mathbb{R}$, on the compact time interval $[0,2]$ we 
can change to an equivalent probability measure $P^{x}$ so that under $P^{x}$ the process $X_{t}=W_{t}+x t$ becomes a standard Brownian motion with no drift, on $[0,1]$. By Girsanov's theorem we have that $P^{x}$ given by

$$
\frac{d P^{x}}{d P^{*}}=\mathcal{E}(-x W)_{1}
$$

satisfies the desired property. We next define a $\mathbb{G}$ stopping time $\tau$ by

$$
\tau=\inf \left\{t:\left|W_{t}\right| \geq 1\right\} \wedge 1
$$

and

$$
f(x)=E^{P^{*}}\left[W_{\tau} \mathcal{E}\left(-x W_{\tau}\right)\right]=E^{P^{*}}\left[W_{\tau} \exp \left\{-x W_{\tau}-\frac{x^{2}}{2} \tau\right\}\right]=E^{P^{x}}\left[W_{\tau}\right]
$$

Since $X$ is a standard Brownian motion on $[0,1]$ under $P^{x}$ and $\tau$ is a stopping time bounded by $1, E^{P^{x}}\left[X_{\tau}\right]=0 ;$ but

$$
0=E^{P^{x}}\left[X_{\tau}\right]=E^{P^{x}}\left[W_{\tau}+x \tau\right]=E^{P^{x}}\left[W_{\tau}\right]+x E^{P^{x}}[\tau]=f(x)+x E^{P^{x}}[\tau]
$$

whence $f(x)=-x E^{P^{x}}[\tau]$. Since $\left|W_{\tau}\right|$ is bounded by 1 and $\tau$ strictly positive a.s. we know $-1<f(x)<0$ for all $x>0$. By Lebesgue's dominated convergence theorem we have that $f$ is continuous on $\mathbb{R}_{+}$. Also, $f(0)=0$. Therefore $f([0,1])=[-R, 0]$ for some constant $R \in(0,1)$.

Recall we are working under the filtration $\mathbb{G}$, relative to which $W$ is a standard Brownian motion under $P^{*}$. We define two stopping times $\rho$ and $\tilde{\tau}$ as follows.

$$
\rho=\inf \left\{t:\left|W_{t}\right| \geq \frac{R}{2}\right\} \wedge 1
$$

and

$$
\tilde{\tau}=\inf \left\{t \geq 1:\left|W_{t}-W_{1}\right| \geq 1\right\} \wedge 2
$$

Define

$$
S_{t}=2+W_{t \wedge \rho}+\left(W_{t \wedge \tilde{\tau}}-W_{1}\right) 1_{[1,2]}(t)>0 .
$$

Finally, we let $\mathbb{F}:=\left(\mathcal{F}_{t}\right)$ denote the filtration generated by $S$. We are only interested in $\left(\mathcal{F}_{t}\right)_{0 \leq t \leq 2}$.

Note that $\rho<\tau$ and $\rho<\tilde{\tau}$ as well. If we let $\mathcal{H}_{t}=\sigma\left\{W_{s}-W_{1} ; 1 \leq s \leq t\right\}$ then the filtration $\mathbb{H}=\left(\mathcal{H}_{t}\right)_{t \geq 1}$ is independent of $\mathcal{F}_{1}$ by the independent increments of $W$ under $P^{*}$. Moreover if 
$\tilde{W}_{t}=W_{t}-W_{1}$ for $t \geq 1$, then $\left(\tilde{W}_{t}, \mathbb{H}\right)_{t \geq 1}$ is a Brownian motion independent from $\left(W_{t \wedge 1}\right)$.

We can also write $S$ as a stochastic integral: $S_{t}-2=\int_{0}^{t} H_{s} d W_{s}$ where $H_{t}=1_{[0, \rho]}(t)+1_{(1, \tilde{\tau}]}(t)$; then $S$ is at least a $P^{*}$-local martingale, but also $S$ is a bounded process and hence it is a $P^{*}$ martingale. Note further that $S$ has the same intervals of constancy as $t \mapsto[S, S]_{t}$ and hence is constant on the random intervals $[\rho, 1]$ and $[\tilde{\tau}, 2]$.

We are now ready to show that $(S, \mathbb{F})$ has martingale representation for $[0,2]$. We first need to show that $S$ is martingale for its own filtration $\mathbb{F}$. However since martingales for an arbitrary filtration to which they are adapted remain martingales for their own filtration, we only need to show $S$ is a martingale for a larger filtration, and this we have already done. To show $S$ has martingale representation, we can invoke the Second Fundamental Theorem of Asset Pricing. This theorem states that $(S, \mathbb{F})$ has the martingale representation property on $[0,2]$ if for any other measure $Q$ equivalent to $P^{*}$ on $\mathcal{F}_{2}$ such that $(S, \mathbb{F})$ is a $Q$-local martingale, we have that $Q=P^{*}$ on $\mathcal{F}_{2}$. This result essentially corresponds to Corollary 11.4 in Jacod (1979) (see also Harrison and Pliska (1983) and Section 9.5 of Chesney et al. (2009)). Let $Q$ be a probability measure equivalent to $\mathrm{P}$ on $\mathcal{F}_{2}$ such that $(S, \mathbb{F})$ is a $Q$-local martingale. Then since the quadratic variation of $S$ is $[S, S]_{t}=\int_{0}^{t}\left(1_{[0, \rho]}(s)+1_{(1, \tilde{\tau}]}(s)\right) d s$, by Lévy's theorem, $\left(S_{t}-S_{0}\right)$ is a Brownian motion on the stochastic interval $[0, \rho]$ with respect to $Q$ and $\left(\mathcal{F}_{t}\right)_{0 \leq t \leq 1}$, and $\left(S_{t}-S_{1}\right)$ is also a Brownian on the interval $[1, \tilde{\tau}]$ with respect to $Q$ and $\left(\mathcal{H}_{t}\right)_{1 \leq t \leq 2}$ which is independent of $\mathcal{F}_{1}$. Additionally, $S$ is constant on the intervals $[\rho, 1]$ and $[\tilde{\tau}, 2]$. Therefore under $Q$ the process $S$ has exactly the same distribution as it does for $P^{*}$ unless $\rho$ and/or $\tilde{\tau}$ have different distributions for $Q$ and $P^{*}$. Now, $\rho$ can be defined in terms of the paths of $S$ before $\rho$, so it will have the same distribution under both $P^{*}$ and $Q$, since $S$ is a Brownian motion for each time before $\rho$ due to Lévy's theorem. And $\tilde{\tau}$ can be defined in terms of the paths of $S$ after 1 up to $\tilde{\tau}$, and since again $\left(S_{t}-S_{1}\right)_{t \geq 1}$ behaves like a standard Brownian motion for both $Q$ and $P^{*}$, the distribution of $\tilde{\tau}$ is the same under both $P^{*}$ and $Q$. Therefore $Q=P^{*}$ on $\mathcal{F}_{2}$ and since $Q$ was arbitrarily chosen by the Second Fundamental Theorem of Asset Pricing we have that $(S, \mathbb{F})$ has martingale representation on $[0,2]$.

We now turn our attention to the futures process $F=\left(F_{t}\right)_{t \geq 0}$. First, for $y \in[-R, 0]$ define

$$
\mu(y)=\inf \{x \geq 0: f(x)=y\}
$$

and $Q^{*}$ on $\mathcal{F}_{2}$ by

$$
\frac{d Q^{*}}{\left.d P^{*}\right|_{\mathcal{F}_{2}}}=\mathcal{E}\left(-\int_{1}^{2} \mu\left(-R / 2-S_{1}+2\right) d S_{s}\right)
$$


Notice that $Q^{*}$ is well-defined because the function $\mu$ is bounded on $[-R, 0]$ by 1 . Intuitively, the measure $Q^{*}$ gives drift to the paths of $S$ according to their level at time 1 . We have by Bayes' formula, the tower property for conditional expectations, the independence lemma for conditional expectations, and the Markov property of the Brownian motion $W$ that

$$
\begin{aligned}
& E^{Q^{*}}\left[S_{2}-S_{1} \mid \mathcal{F}_{1}\right]=E^{Q^{*}}\left[W_{\tilde{\tau}}-W_{1} \mid \mathcal{F}_{1}\right] \\
& =E^{P^{*}}\left[E^{P^{*}}\left[\left(W_{\tilde{\tau}}-W_{1}\right) \exp \left\{-\mu\left(-R / 2-S_{1}+2\right)\left(W_{\tilde{\tau}}-W_{1}\right)-\frac{\left(\mu\left(-R / 2-S_{1}+2\right)\right)^{2}}{2}(\tilde{\tau}-1)\right\} \mid \mathcal{G}_{1}\right] \mid \mathcal{F}_{1}\right] \\
& =f\left(\mu\left(-R / 2-S_{1}+2\right)\right) \\
& =-R / 2-S_{1}+2 .
\end{aligned}
$$

Therefore

$$
E^{Q^{*}}\left[S_{2} \mid \mathcal{F}_{t}\right]=E^{Q^{*}}\left[S_{2}-S_{1} \mid \mathcal{F}_{t}\right]+S_{1} \equiv 2-R / 2 \text { for } t \leq 1
$$

$S$ is a $\left(Q^{*}, \mathbb{F}\right)$-supermartingale $\left(\mu\left(-R / 2-S_{1}+2\right) \geq 0\right)$ and

$$
F_{t}:=E^{Q^{*}}\left[S_{2} \mid \mathcal{F}_{t}\right]
$$

Note that $F$ is a $Q^{*}$ martingale, being the projection of $S_{2}$ onto a filtration $\mathbb{F}$, but the filtration $\mathbb{F}$ is not constant on $[0,1]$ while $F$ is constant there. Since any stochastic integral with respect to $F$ must also be constant where $F$ is constant, $(F, \mathbb{F})$ does not have martingale representation for $Q^{*}$.

In the Brownian framework we can obtain inter alia necessary and sufficient conditions for market completeness to be inherited by the futures price process, albeit within the confines of a heavily hypothesized paradigm.

Example 6.10 Suppose that $d S_{t}=S_{t}\left(\mu d t+\sigma d B_{t}\right)$ with $B$ a P-Brownian motion and let $Q^{*} \in$ $\mathcal{M}_{\text {sup }}(S)$ be as in Example 4.5. By following the steps of Example 4.5 we can prove that

$$
F_{t, T}=S_{0} E^{Q^{*}}\left[\exp \left(\int_{0}^{T}\left(\mu+\sigma \eta_{s}\right) d s\right) \mathcal{E}\left(\sigma B^{*}\right)_{T} \mid \mathcal{F}_{t}\right]
$$

where $B^{*}$ is a $Q^{*}$-martingale with respect to $\mathbb{F}$ and $\eta$ is a predictable process such that $(\mu+\sigma \eta) \leq 0$ $P \otimes \lambda$-almost surely, where $\lambda$ is Lebesgue measure on $[0, T]$.

Assume that $\mathbb{F}$ is the minimal filtration generated by $B^{*}$ (this is a rather delicate assumption, and an interesting discussion on this subject can be found in Chapter V of Revuz and Yor (1999) 
and Section 5.7.1 of Chesney et al. (2009)). Assume that $\mu+\sigma \eta-\frac{\sigma^{2}}{2}=-k\left(B_{t}^{*}\right)$ for some continuous and nonnegative function $k$ (this hypothesis is also quite delicate since we are imposing a particular functional form dependence of $\eta_{t}$ on $B_{t}$ and $\eta_{s}$ for $s<t$ ).

We can then write

$$
\begin{aligned}
F_{t, T} & =S_{0} E^{Q^{*}}\left[\exp \left(-\int_{0}^{T} k\left(B_{s}^{*}\right) d s\right) \exp \left(\sigma B_{T}^{*}\right) \mid \mathcal{F}_{t}\right] \\
& =S_{0} \exp \left(-\int_{0}^{t} k\left(B_{s}^{*}\right) d s\right) E^{Q^{*}}\left[\exp \left(-\int_{t}^{T} k\left(B_{s}^{*}\right) d t\right) \exp \left(\sigma B_{T}^{*}\right) \mid \mathcal{F}_{t}\right]
\end{aligned}
$$

Using the Markovian property of $B^{*}$ with respect to $\mathcal{F}_{t}$ and assuming that the hypotheses of the Theorem of Feynman-Kac (Theorem 4.4.2 in Karatzas and Shreve (1998a)) hold then we can write

$$
F_{t, T}=S_{0} \exp \left(-\int_{0}^{t} k\left(B_{s}^{*}\right) d s\right) v\left(t, B_{t}^{*}\right)
$$

where

$$
v(t, x)=E^{x}\left[\exp \left(-\int_{0}^{T-t} k\left(B_{s}^{*}\right) d s\right) \exp \left(\sigma B_{T-t}^{*}\right)\right]
$$

solves the Cauchy-problem

$$
\begin{array}{r}
-\frac{\partial v}{\partial t}+k v=\frac{1}{2} \Delta v ; \quad \text { on }[0, T) \times \mathbb{R} \\
v(T, x)=\exp (\sigma x) ; \quad x \in \mathbb{R} .
\end{array}
$$

By Itô's formula

$$
d F_{t, T}=S_{0} \exp \left(-\int_{0}^{t} k\left(B_{s}^{*}\right) d s\right) v_{x}\left(t, B_{t}^{*}\right) d B_{t}^{*},
$$

where $v_{x}$ denotes the first partial derivative of $v$ is the $x$ argument.

We would have in this case that the futures market is complete under $P^{*}$ (for the original filtration $\mathbb{F})$ if and only if the process $\left(v_{x}\left(t, B_{t}^{*}\right)\right)_{0 \leq t \leq T}$ is nonzero $P \otimes \lambda$-almost surely, where $\lambda$ is Lebesgue measure on $[0, T]$.

An alternative way to describe the dynamics of the futures price process when the process $\eta$ is not deterministic uses Malliavin Calculus. Under technical assumptions on $\eta$, by the Generalized 
Clark-Ocone formula (see Karatzas and Ocone (1991)) we have that

$$
d F_{t, T}=\left(E^{Q^{*}}\left[D_{t} F \mid \mathcal{F}_{t}\right]+E^{Q^{*}}\left[F \int_{t}^{T} D_{t} \eta_{u} d B_{u}^{*} \mid \mathcal{F}_{t}\right]\right) d B_{t}^{*}
$$

where $F=S_{T}=S_{0} \exp \left(\mu T+\sigma B_{T}-\frac{\sigma^{2}}{2} T\right)$ and $D_{t}$ denotes the Malliavin derivative. By using properties of the Malliavin derivative it is straightforward to see that

$$
D_{t} F=\sigma F
$$

This implies that

$$
d F_{t, T}=\left(\sigma F_{t, T}+E^{Q^{*}}\left[S_{T} \int_{t}^{T} D_{t} \eta_{u} d B_{u}^{*} \mid \mathcal{F}_{t}\right]\right) d B_{t}^{*}
$$

When $\eta$ is deterministic this yields

$$
d F_{t, T}=\sigma F_{t, T} d B_{t}^{*}
$$

and $F_{t, T}=F_{0, T} \mathcal{E}\left(\sigma B^{*}\right)_{t}=S_{0} \exp \left(\int_{0}^{T}\left(\mu+\sigma \eta_{s}\right) d s\right) \mathcal{E}\left(\sigma B^{*}\right)_{t}$, as already observed in Example 4.5.

However, when $\eta$ is random, the volatility of the process $F_{t, T}$ is not necessarily equal to $\sigma$ and has an additional term equal to

$$
E^{Q^{*}}\left[S_{T} \int_{t}^{T} D_{t} \eta_{u} d B_{u}^{*} \mid \mathcal{F}_{t}\right]
$$

This difference in volatility in markets with short sales prohibition was experimentally confirmed in Charoenrook and Daouk (2005). Also observe that the futures market is complete if and only if

$$
\left(\sigma F_{t, T}+E^{Q^{*}}\left[S_{T} \int_{t}^{T} D_{t} \eta_{u} d B_{u}^{*} \mid \mathcal{F}_{t}\right]\right) \neq 0
$$

Lebesgue-almost everywhere $t \in[0, T]$, P-almost surely.

Under the hypothesis of this example, expression (6.3) provides necessary and sufficient conditions for the futures market to inherit the completeness property from the underlying asset price process. Essentially, the volatility of the futures price process must not be zero for any open time interval over the futures contract's maturity. If the volatility is zero, then the futures price does not reflect the randomness generating the underlying asset price process. And of course, the futures 
market would not be complete.

\section{STOCHASTIC INTEREST RATES}

In the previous sections the spot rate of interest was equal to zero, and futures and forward prices coincided (see Remark 5.3). It is well known that without short sales restrictions or dividend payments, futures prices differ from forward prices by a factor depending on interest rates (see for instance Duffie (1989) and Jarrow and Protter (2009)). We have showed that with short sale prohibitions, when the overpricing hypothesis holds, and when the spot rate of interest is zero, there is a difference between the asset price process and the futures price process originating from the multiplicative decomposition of the underlying asset price process with respect to the market's pricing measure $Q^{*}$ (see Theorem 5.2). Hence, in a market with: (1) short sale prohibitions, (2) stochastic interest rates, (3) when the overpricing hypothesis holds, and (4) when the spot price process has a bubble, then the difference between futures and the spot asset prices can be expressed as a combination of two factors, the one relating to interest rates and the aforementioned "short sales prohibition discount factor". In this section we exhibit explicit formulas for this difference. We have the following extension of Theorem 5.2.

Theorem 7.1 Suppose that $S>0$ and Assumption 4.4 holds. Suppose that the canonical DoobMeyer decomposition of the $Q^{*}$-supermartingale $\tilde{S}$ is $\tilde{S}=\tilde{S}_{0}+\tilde{N}+\tilde{V}$ and that the multiplicative decomposition of $\tilde{S}$ under $Q^{*}$ is $\tilde{S}=\tilde{L} \tilde{D}$. Where

$$
\begin{gathered}
\tilde{L}=\mathcal{E}\left(\left(\frac{1}{\tilde{S}_{-}+\Delta \tilde{V}}\right) \cdot \tilde{N}\right) \\
\tilde{D}=\tilde{S}_{0}\left(\mathcal{E}\left(-\left(\frac{1}{\tilde{S}_{-}+\Delta \tilde{V}}\right) \cdot \tilde{V}\right)\right)^{-1} .
\end{gathered}
$$

Then the following holds.

1. If $\tilde{N}$ is a $Q^{*}$-martingale and $\tilde{V}_{T}, S_{T}^{0} \in \mathcal{F}_{t_{0}}$ for some $t_{0}<T$ then $F_{t, T}=S_{0}^{T}\left(\tilde{S}_{0}+\tilde{N}_{t}+\tilde{V}_{T}\right)$ for all $t \in\left[t_{0}, T\right]$.

2. If $\tilde{L}$ is a $Q^{*}$-martingale and $\tilde{D}_{T}, S_{T}^{0} \in \mathcal{F}_{t_{0}}$ for some $t_{0}<T$, then $F_{t, T}=L_{t} S_{T}^{0} D_{T}$ for all $t \in\left[t_{0}, T\right]$. 
Proof. This theorem follows directly from

$$
F_{t, T}=E^{Q^{*}}\left[S_{T} \mid \mathcal{F}_{t}\right]=S_{T}^{0} E^{Q^{*}}\left[\tilde{S}_{T} \mid \mathcal{F}_{t}\right] \text { for } t \geq t_{0}
$$

When the assumptions on $S$ in the previous theorem do not hold, then there is an alternative way to represent the futures price process using additional assumptions on the stochastic evolution of the term structure of interest rates. Define

$$
p(t, T):=E^{Q^{*}}\left[\left(S_{T}^{0}\right)^{-1} \mid \mathcal{F}_{t}\right]
$$

to be the (discounted) price at time $t$ of a zero-coupon bond with maturity $T$. We have the following alternative characterization of the futures price process

Theorem 7.2 With the notation of Theorem 7.1 and under Assumption 4.4, let

$$
\begin{gathered}
X:=\left(\frac{1}{\tilde{S}_{-}+\Delta \tilde{V}}\right) \cdot \tilde{N}, \\
R:=-\left(\frac{1}{\tilde{S}_{-}+\Delta \tilde{V}}\right) \cdot \tilde{V},
\end{gathered}
$$

and suppose that

$$
p(\cdot, T)=p(0, T) \mathcal{E}(Y)
$$

for a $Q^{*}$-local martingale $Y$ with $\Delta Y>-1$. Then, the futures price process is given by

$$
F_{t, T}=\frac{\tilde{S}_{0}}{p(0, T)} E^{Q^{*}}\left[\frac{\mathcal{E}(Z)_{T}}{\mathcal{E}(R)_{T}} \mid \mathcal{F}_{t}\right]
$$

where

$$
Z:=X-Y-\left[X^{c}-Y^{c}, Y^{c}\right]-\sum_{s \leq .}\left(\Delta(X-Y)_{s} \frac{\Delta Y_{s}}{1+\Delta Y_{s}}\right)
$$

and $X^{c}, Y^{c}$ are the continuous parts of the $Q^{*}$-local martingales $X$ and $Y$, respectively. 
Proof. Observe that by Lemma 5.1 and Theorem 7.1

$$
\begin{aligned}
F_{t, T} & =E^{Q^{*}}\left[S_{T} \mid \mathcal{F}_{t}\right] \\
& =E^{Q^{*}}\left[\tilde{S}_{T}\left(S_{T}^{0}\right) \mid \mathcal{F}_{t}\right] \\
& =E^{Q^{*}}\left[\frac{\tilde{S}_{T}}{p(T, T)} \mid \mathcal{F}_{t}\right] \\
& =\frac{\tilde{S}_{0}}{p(0, T)} E^{Q^{*}}\left[\frac{\mathcal{E}(X)_{T}}{\mathcal{E}(Y)_{T} \mathcal{E}(R)_{T}} \mid \mathcal{F}_{t}\right] .
\end{aligned}
$$

The theorem follows from Lemma 3.4 in Karatzas and Kardaras (2007), which shows that

$$
\frac{\mathcal{E}(X)}{\mathcal{E}(Y)}=\mathcal{E}(Z) .
$$

Remark 7.3 The ideas presented in this theorem extend those in Amin and Jarrow (1992). In their work, there is no short sales prohibition (so $R \equiv 0$ ), the processes $X$ and $Y$ are continuous, $[X-Y, Y]$ is assumed to be deterministic, and $\mathcal{E}(X-Y)$ is a $Q^{*}$-martingale. In this case we can rewrite formula (7.2) as

$$
\begin{aligned}
F_{t, T} & =\frac{\tilde{S}_{0} \exp \left(-[X-Y, Y]_{T}\right)}{p(0, T)} \mathcal{E}(X-Y)_{t} \\
& =\frac{\tilde{S}_{0} \exp \left(-[X-Y, Y]_{T}+[X-Y, Y]_{t}\right)}{p(0, T)} \mathcal{E}(Z)_{t} \\
& =\frac{\tilde{S}_{t}}{p(t, T)} \exp \left(-[X-Y, Y]_{T}+[X-Y, Y]_{t}\right),
\end{aligned}
$$

which corresponds to equation (3.26) in Amin and Jarrow (1992).

These results exhibit explicitly the fact that the discount factors of futures prices have two sources of randomness, $X$ and $Y$, one coming from the underlying asset price process and another from stochastic interest rates $Y$. Hence, in order to use futures contracts to hedge the asset's price randomness, it is not only important to adjust for the "short sales prohibition discount factor" $\mathcal{E}(R)^{-1}$, but also for the interest rate factor as well. Traded zero-coupon bonds are needed to hedge this later randomness. 


\section{THE MULTI-DIMENSIONAL CASE AND FUTURES ON AN INDEX}

This section presents the natural extension of the previous results to multidimensional markets. We also study the behavior of futures on an index, and the effect of short sale prohibitions on hedging strategies using these instruments. These results are of particular importance for assets that cannot be short sold, where futures on the individual asset are impractical, but where futures on an index of the assets (a portfolio) can be traded. The two prime examples satisfying these conditions are residential homes, and individual equities with short sale constraints. Another example would be for electricity and electricity futures, where the underlying commodity - electricity - cannot be stored in significant quantities.

We again adopt the notation used in Section 2. We assume that there are $N$ securities trading in the market, from which the first $d$ assets can be sold short in an admissible way (see Definition 3.1) and the last $N-d$ cannot. Let $S=\left(S^{1}, \ldots, S^{N}\right)$ represent the price processes of these assets. In this section, to simplify our notation we will assume again that the spot rate of interest is identically equal to zero, however we note that the results can be extended to the case of stochastic interest rates using the ideas from the previous section.

Throughout this section we will assume that $S^{i}>0$ for all $i \leq N$. For each $i$, the Doob-Meyer decomposition of the $Q^{*}$-supermartingale $S^{i}$ is $S^{i}=S_{0}^{i}+N^{i}+V^{i}$. For each $i$, we denote by $L^{i}$ and $D^{i}$ the local martingale and predictable part, respectively, in the multiplicative decomposition $S^{i}=L^{i} D^{i}$. For each $i \leq N$ we suppose that Assumption 4.4 holds for the futures price of a futures contract on $S^{i}$ with maturity $T$. In other words, we assume that if $F_{\cdot, T}^{i}$ is the futures price of a futures contract on $S^{i}$ with maturity $T$ then

\section{Assumption 8.1}

$$
F_{\cdot, T}^{i} \text { is a } Q^{*} \text {-martingale. }
$$

We denote by $F .=\left(F_{\cdot, T}^{1}, \ldots, F_{\cdot, T}^{N}\right)$ the vector of futures price processes. For fixed deterministic positive weights $\omega_{i}$ we define the index value by

$$
I=\sum_{i=1}^{N} \omega_{i} S^{i}
$$

The following observation immediately follows. 
Proposition 8.2 If $I$ is a $Q^{*}$-martingale, then for all $i \leq N, S^{i}$ is a $Q^{*}$-martingale.

Proof. Since $S^{i}$ is a $Q^{*}$-supermartingale for all $i \leq N$ we have that if $E^{Q^{*}}\left[S_{T}^{i} \mid \mathcal{F}_{i}\right]<S_{t}^{i}$ for some $i \leq N$ and $t<T$ then

$$
I_{t}=E^{Q^{*}}\left[I_{T} \mid \mathcal{F}_{t}\right]=\sum_{i \leq N} \omega_{i} E^{Q^{*}}\left[S_{T}^{i} \mid \mathcal{F}_{t}\right]<\sum_{i \leq N} \omega_{i} S_{t}^{i}=I_{t}
$$

which is a contradiction. Then, it must be that $S^{i}$ is a $Q^{*}$-martingale for all $i \leq N$.

This proposition shows that if the index $I$ is traded, sold short, and has no bubble then none of the underlying asset price processes have bubbles either, using the definiton of bubbles in Jarrow et al. (2007), Jarrow et al. (2010).

It is also of interest to study futures contracts on indexes. In this regard we make the following assumption.

\section{Assumption 8.3}

The futures price of a futures contract on $I, F_{\cdot, T}^{I}$, is a $Q^{*}$-martingale.

The following theorem describes the behavior of futures on an index and a related hedging result.

Theorem 8.4 Assume that for all $i \leq N D^{i}$ is deterministic and $L^{i}$ is a true $Q^{*}$-martingale and that Assumptions 8.1 and 8.3 hold. We have that if for some probability measure $P^{*} \sim P$

$$
\mathcal{M}_{\text {loc }}(S)=\left\{Q \sim P: S^{i} \text { is a } Q \text {-local martingale for all } i \leq N\right\}=\left\{P^{*}\right\}
$$

then,

$$
\mathcal{M}_{\text {loc }}(F):=\left\{Q \sim P: F_{, T}^{i} \text { is a } Q \text {-local martingale for all } i \leq N\right\}=\left\{Q^{*}\right\} .
$$

Additionally, any $Q^{*}$-local martingale $M$ can be written as

$$
M=M_{0}+(H \cdot F),
$$

for some predictable process $H \in L(F)$, where $L(F)$ is the space of predictable processes integrable with respect to $F$. 
If additionally $H^{i} \in L\left(F_{\cdot, T}^{i}\right)$ for all $i \leq N$ then

$$
M=M_{0}+(K \cdot Y),
$$

where $Y=\left(F_{\cdot, T}^{1}, \ldots, F_{\cdot, T}^{N}, F^{I}\right)$ and $K$ is a predictable process in $L(Y)$ such that $K^{i} \geq 0$ for all $i \leq N$.

Proof. That $\mathcal{M}_{l o c}(F)=\left\{Q^{*}\right\}$ and that any $Q^{*}$-local martingale $M$ can be written as

$$
M=M_{0}+(H \cdot F),
$$

for some predictable process $H \in L(F)$, follows from the Second Fundamental Theorem of Asset pricing (see Corollary 11.4 in Jacod (1979), Harrison and Pliska (1983) and Section 9.5 of Chesney et al. (2009))) and Theorem 6.3 (the same proofs apply to this framework). It remains to prove the representation property with respect to the futures contract on $I$. Let $M$ be an arbitrary $Q^{*}$-martingale and $H \in L(F)$ such that

$$
M=M_{0}+(H \cdot M)
$$

We have that for each $i \leq N$

$$
-d F_{t, T}^{i}=\frac{1}{\omega_{i}}\left(-d F_{t}^{I}+\sum_{j \leq N, j \neq i} \omega_{j} d F_{t, T}^{j}\right) .
$$

If additionally $H^{i} \in L\left(F_{\cdot, T}^{i}\right)$ for all $i \leq N$, we can write (see Jacod (1980))

$$
\begin{aligned}
H_{t} d F_{t} & =\sum_{i \leq N} H_{t}^{i} d F_{t, T}^{i} \\
& =\sum_{i \leq N}\left(H_{t}^{i} 1_{\left\{H_{t}^{i} \geq 0\right\}} d F_{t, T}^{i}+H_{t}^{i} 1_{\left\{H_{t}^{i}<0\right\}} d F_{t, T}^{i}\right) \\
& =\sum_{i \leq N}\left(H_{t}^{i} 1_{\left\{H_{t}^{i} \geq 0\right\}} d F_{t, T}^{i}+\left(-H_{t}^{i}\right) 1_{\left\{H_{t}^{i}<0\right\}}\left(-d F_{t, T}^{i}\right)\right) \\
& =\sum_{i \leq N}\left(H_{t}^{i} 1_{\left\{H_{t}^{i} \geq 0\right\}} d F_{t, T}^{i}+\left(-H_{t}^{i}\right) 1_{\left\{H_{t}^{i}<0\right\}} \frac{1}{\omega_{i}}\left(-d F_{t, T}^{I}+\sum_{j \leq N, j \neq i} \omega_{j} d F_{t, T}^{j}\right)\right) .
\end{aligned}
$$


Observe that the last equation can be rewritten as

$$
H_{t} d F_{t}=\sum_{i \leq N} K_{t}^{i} d F_{t, T}^{i}+K_{t}^{N+1} d F_{t, T}^{I}
$$

with $K^{i} \geq 0$ for all $i \leq N$.

Remark 8.5 This theorem states that under certain technical assumptions, hedging strategies involving all the futures contracts on the asset price processes can be replaced by a trading strategy using a futures on an index and trades that are only long on these futures contracts.

It is also important to note that in order to hedge claims using the futures contract on an index, one needs the futures contracts on the individual asset price processes, rather than the asset price processes themselves. A short position on $F_{\cdot, T}^{I}$ combined with a long position on the individual asset price processes (modulus some constant coefficients) is not necessarily equivalent to a short position on the asset price process due to the factors $D^{i}$ by which asset and futures prices differ (see equation (8.2)). More precisely, we have that

$$
d F_{t, T}^{i}=D_{T} d L_{t}^{i}
$$

while

$$
d S_{t}^{i}=D_{t-}^{i} d L_{t}^{i}+L_{t-}^{i} d D_{t}^{i}+d\left[D_{t}^{i}, L_{t}^{i}\right]
$$

Nonetheless, we see how the difference between the dynamics of the futures and asset price processes depends on the "overpricing discount factor" D.

\section{A SEMIMARTINGALE CHARACTERISTICS UNDER THE REFERENCE MEASURE}

For a more detailed discussion of the results presented below we refer the reader to Chapter III of Jacod and Shiryaev (2003). To simplify our notation, in this appendix we will assume that $S^{0} \equiv 1$ and there are no cash flows. However under mild hypotheses, by the results presented in the Section 3 (see Theorem 3.2), the analysis below can be extended to markets with stochastic interest rates and assets with cash flows. In order to obtain such an extension, one replaces the underlying asset price process by the discounted process plus its discounted cash flows. 
Let $S$ be an $\mathbb{R}^{N}$-valued process representing the prices of the risky assets in the market. Since we have assumed that $S$ is a $P$-semimartingale, it has a canonical representation given by

$$
S=S_{0}+S^{c}+\left(x 1_{\{|x| \leq 1\}}\right) *\left(\mu^{S}-\nu\right)+\left(x 1_{\{|x|>1\}}\right) * \mu^{S}+B
$$

where $*$ denotes integration with respect to a random measure (see Section II-1a of Jacod and Shiryaev (2003)) and,

(i) $S^{c}$ is a continuous $P$-local martingale starting at 0 , known as the continuous martingale part of $\mathrm{S}$,

(ii) $\mu^{S}$ is the random measure associated to the jumps of $S$ defined by

$$
\mu^{S}([0, t] \times A)=\sum_{s \leq t} 1_{A \backslash\{0\}}\left(\Delta S_{s}\right)
$$

for $0 \leq t \leq T$ and $A \subset \mathbb{R}^{N}$,

(iii) $\nu$ is the compensator of the random measure $\mu^{S}$ (see Theorem II-1.8 in Jacod and Shiryaev $(2003))$,

(iv) $B$ is a predictable $\mathbb{R}^{N}$-valued process with components of finite variation.

If we define $C_{i, j}=\left[\left(S^{c}\right)^{i},\left(S^{c}\right)^{j}\right]$ then $(B, C, \nu)$ are known as the semimartingale characteristics of $S$ under $P$ with respect to the canonical truncation function $h(x)=x 1_{\{|x| \leq 1\}}$. According to Proposition II-2..9 in Jacod and Shiryaev (2003) one can find a version of the characteristics $(B, C, \nu)$ of $S$ of the form

$$
\begin{aligned}
& B=b \cdot A, \\
& C=c \cdot A \\
& \nu(\omega, d t, d x)=d A_{t}(\omega) K_{\omega, t}(d x),
\end{aligned}
$$

where $A$ is a predictable locally integrable nondecreasing process; $b$ and $c$ are predictable processes, with $b$ taking values in $\mathbb{R}^{N}$ and $c$ taking values in the set of symmetric nonnegative $N \times N$ matrices 
and $K_{\omega, t}(d x)$ is a transition kernel from $(\Omega \times[0, T], \mathcal{P})$ into $\left(\mathbb{R}^{N}, \mathcal{B}\left(\mathbb{R}^{N}\right)\right)$ which satisfies

$$
\begin{aligned}
& K_{\omega, t}(\{0\})=0, \int K_{\omega, t}(d x)\left(|x|^{2} \wedge 1\right) \leq 1, \\
& \Delta A_{t}(\omega)>0 \Rightarrow b_{t}(\omega)=\int K_{\omega, t}(d x) x 1_{\{|x \leq 1|\}}, \\
& \Delta A_{t}(\omega) K_{\omega, t}\left(\mathbb{R}^{N}\right) \leq 1 .
\end{aligned}
$$

\section{B SEMIMARTINGALE CHARACTERISTICS UNDER THE PRICING MEASURE}

Now, given the measure $Q^{*}$ (see Assumption 4.4), Girsanov's Theorem for semimartingales (Theorem III-3.24 in Jacod and Shiryaev (2003)) implies that there exists a nonnegative $\tilde{\mathcal{P}}:=\mathcal{P} \otimes \mathcal{B}\left(\mathbb{R}^{N}\right)$ measurable function $Y^{*}$ (where $\mathcal{B}\left(\mathbb{R}^{N}\right)$ is the Borel sigma-algebra on $\mathbb{R}^{N}$ and $\otimes$ denotes the product sigma-algebra) and a predictable process $\beta^{*}$ satisfying

$$
\begin{gathered}
\left|x 1_{\{|x| \leq 1\}}\left(Y^{*}-1\right)\right| * \nu_{t}<\infty Q^{*} \text {-almost surely for all } t \in[0, T] \\
\left|\sum_{j \leq N} c^{i j} \beta^{*, j}\right| \cdot A_{t}<\infty \text { and }\left(\sum_{j, k \leq N} \beta^{*, j} c^{j k} \beta^{*, k}\right) \cdot A_{t}<\infty Q \text {-almost surely for all } i \text { and } t \in[0, T] \\
\nu(\omega ;\{t\} \times E)=1 \Rightarrow \int Y^{*}(\omega, t, x) \nu(\omega ;\{t\} \times d x)=1
\end{gathered}
$$

and such that the characteristics of $S$ relative to $Q^{*}$ are

$$
\begin{aligned}
& B^{*}=B+c \beta^{*}+x 1_{\{|x| \leq 1\}}\left(Y^{*}-1\right) * \nu, \\
& C^{*}=C, \\
& \nu^{*}=Y^{*} \cdot \nu, \text { where } Y^{*} \cdot \nu(\omega ; d t, d x)=\nu(\omega ; d t, d x) Y^{*}(\omega, t, x) .
\end{aligned}
$$

Furthermore, according to Lemma III-5.17 in Jacod and Shiryaev (2003), the density process $Z^{*}$ of $Q^{*}$ relative to $P$ has the form

$$
Z^{*}=1+\left(Z_{-}^{*} \beta^{*}\right) \cdot S^{c}+Z_{-}^{*}\left(Y^{*}-1+\frac{\hat{Y}^{*}-a}{1-a} 1_{\{a<1\}}\right) *\left(\mu^{S}-\nu\right)+Z^{\prime}
$$


where

(i) $Z^{\prime}$ is a $P$-local martingale with $Z_{0}^{\prime}=0$ and $\left[\left(Z^{\prime c},\left(S^{i}\right)^{c}\right]=0\right.$ for all $i \leq N$ and $M_{\mu^{S}}^{P}\left[\Delta Z^{\prime} \mid \tilde{\mathcal{P}}\right]=0$ (see III-3.15 in Jacod and Shiryaev (2003)),

(ii)

$$
a_{t}(\omega)=\nu\left(\omega ;\{t\} \times \mathbb{R}^{N}\right)
$$

(iii)

$$
\hat{Y}^{*}{ }_{t}(\omega)= \begin{cases}\int \nu(\omega ;\{t\} \times d x) Y^{*}(\omega, t, x) & \text { if this integral converges } \\ \infty & \text { otherwise. }\end{cases}
$$

\section{References}

Amin, K. I. and Jarrow, R. (1992). Pricing options on risky assets in a stochastic interest rate economy. Mathematical Finance, 2(4):217-237.

Ansel, J. P. and Stricker, C. (1994). Couverture des actifs contingents et prix maximum. Annales de l'Institut Henri Poincaré (B) Probability and Statistics, 30(2):303-315.

Beber, A. and Pagano, M. (2011). Short-selling bans around the world: Evidence from the 2007-09 crisis. Journal of Finance, Forthcoming. Available at SSRN: http://ssrn.com/abstract=1502184.

Boehmer, E., Jones, C. M., and Zhang, X. (2011). Shackling short sellers: The 2008 shorting ban. Working paper, available at SSRN: http://ssrn.com/abstract=1412844.

Boulton, T. J. and Braga Alves, M. V. (2010). The skinny on the 2008 naked short-sale restrictions. Journal of Financial Markets, 13(4):397-421.

Bris, A., Goetzmann, W. N., and Zhu, N. (2007). Efficiency and the bear: Short sales and markets around the world. Journal of Finance, 62(3):1029-1079.

Brown, A. A. and Rogers, L. C. G. (2012). Diverse beliefs. Forthcoming in Stochastics An International Journal of Probability and Stochastic Processes.

Charoenrook, A. and Daouk, H. (2005). A study of market-wide short-selling restrictions. Working paper, available at SSRN: http://ssrn.com/abstract $=687562$.

Chesney, M., Jeanblanc, M., and Yor, M. (2009). Mathematical Methods for Financial Markets. SpringerVerlag.

Cox, A. and Hobson, D. (2005). Local martingales, bubbles and option prices. Finance and Stochastics, 9(4):477-492. 
Cvitanić, J. and Karatzas, I. (1993). Hedging contingent claims with constrained portfolios. The Annals of Applied Probability, 3(3):652-681.

Delbaen, F. and Schachermayer, W. (1994). A general version of the fundamental theorem of asset pricing. Mathematische Annalen, 300(1):463-520.

Delbaen, F. and Schachermayer, W. (1995). Arbitrage possibilities in Bessel processes and their relations to local martingales. Probability Theory and Related Fields, 102(3):357-366.

Duffie, D. (1989). Futures Markets. Prentice Hall.

Ekström, E. and Tysk, J. (2009). Bubbles, convexity and the Black-Scholes equation. Annals of Applied Probability, 19(4):1369-1384.

Fabozzi, F. J. (2004). Short selling: strategies, risks, and rewards. The Frank J. Fabozzi Series. John Wiley \& Sons, Inc.

Frittelli, M. (1997). Semimartingales and asset pricing under constraints. In Mathematics of Derivative Securities (Publications of the Newton Institute), pages 265-277. Cambridge University Press.

Fung, J. K. W. and Draper, P. (1999). Mispricing of index futures contracts and short sales constraints. Journal of Futures Markets, 19(6):695-715.

Harrison, J. M. and Pliska, S. R. (1981). Martingales and stochastic integrals in the theory of continuous trading. Stochastic Processes and their Applications, 11(3):215-260.

Harrison, J. M. and Pliska, S. R. (1983). A stochastic calculus model of continuous trading: Complete markets. Stochastic Processes and their Applications, 15(3):313-316.

Jacod, J. (1979). Calcul stochastique et problèmes de martingales, volume 714 of Lecture Notes in Mathematics. Springer.

Jacod, J. (1980). Intégrales stochastiques par rapport à une semimartingale vectorielle et changements de filtration. Séminaire de Probabilités de Strasbourg, 14:161-172.

Jacod, J. and Shiryaev, A. N. (2003). Limit Theorems for Stochastic Processes. Springer-Verlag, 2nd edition.

Jarrow, R. and Protter, P. (2009). Forward and futures prices with bubbles. International Journal of Theoretical and Applied Finance, 12(7):901-924.

Jarrow, R., Protter, P., and Shimbo, K. (2007). Asset price bubbles in a complete market. In Advances in Mathematical Finance, Applied and Numerical Harmonic Analysis, pages 97-121. Birkhäuser, Boston.

Jarrow, R., Protter, P., and Shimbo, K. (2010). Asset price bubbles in incomplete markets. Mathematical Finance, 20(2):145-185.

Jouini, E. and Kallal, H. (1995). Arbitrage in securities markets with short-sales constraints. Mathematical Finance, 5(3):197-232. 
Karatzas, I. and Kardaras, C. (2007). The numéraire portfolio in semimartingale financial models. Finance and Stochastics, 11(4):447-493.

Karatzas, I. and Ocone, D. (1991). A generalized Clark representation formula, with applications to optimal portfolios. Stochastics, 37:187-220.

Karatzas, I. and Shreve, S. E. (1998a). Brownian Motion and Stochastic Calculus. Springer, 2nd edition.

Karatzas, I. and Shreve, S. E. (1998b). Methods of Mathematical Finance. Springer-Verlag.

Kchia, Y. and Protter, P. (2011). On progressive filtration expansion with a process. arXiv:1105.1662v1 [math.PR].

Madan, D. B. and Yor., M. (2006). Itô's integrated formula for strict local martingales. In In Memoriam Paul-André Meyer, volume 1874 of Lecture Notes in Mathematics, pages 157-170. Springer.

Mijatović, A. and Urusov, M. (2012). On the martingale property of certain local martingales. Probability Theory and Related Fields, 152(1-2):1-30.

Miller, E. M. (1977). Risk, uncertainty and divergence of opinion. Journal of Finance, 32(4):1151-1168.

Napp, C. (2003). The Dalang-Morton-Willinger theorem under cone constraints. Journal of Mathematical Economics, 39(1-2):111-126.

Pal, S. and Protter, P. (2010). Analysis of strict local martingales via h-tranforms. Stochastic Processes and their Applications, 120(8):1424-1443.

Pham, H. and Touzi, N. (1999). The fundamental theorem of asset pricing with cone constraints. Journal of Mathematical Economics, 31(2):265-279.

Protter, P. (2005). Stochastic Integration and Differential Equations. Springer, 2nd edition. Corrected 3rd printing.

Pulido, S. (2011). The fundamental theorem of asset pricing, the hedging problem and maximal claims in financial markets with short sales prohibitions. arXiv:1012.3102v4 [q-fin.PR].

Revuz, D. and Yor, M. (1999). Continuous Martingales and Brownian Motion. Springer, 3rd edition.

Ruf, J. (2012). Hedging under arbitrage. Mathematical Finance. doi: 10.1111/j.1467-9965.2011.00502.x.

Schürger, K. (1996). On the existence of equivalent $\tau$-measures in finite discrete time. Stochastic Processes and their Applications, 61(1):109-128. 


\section{FIGURES}

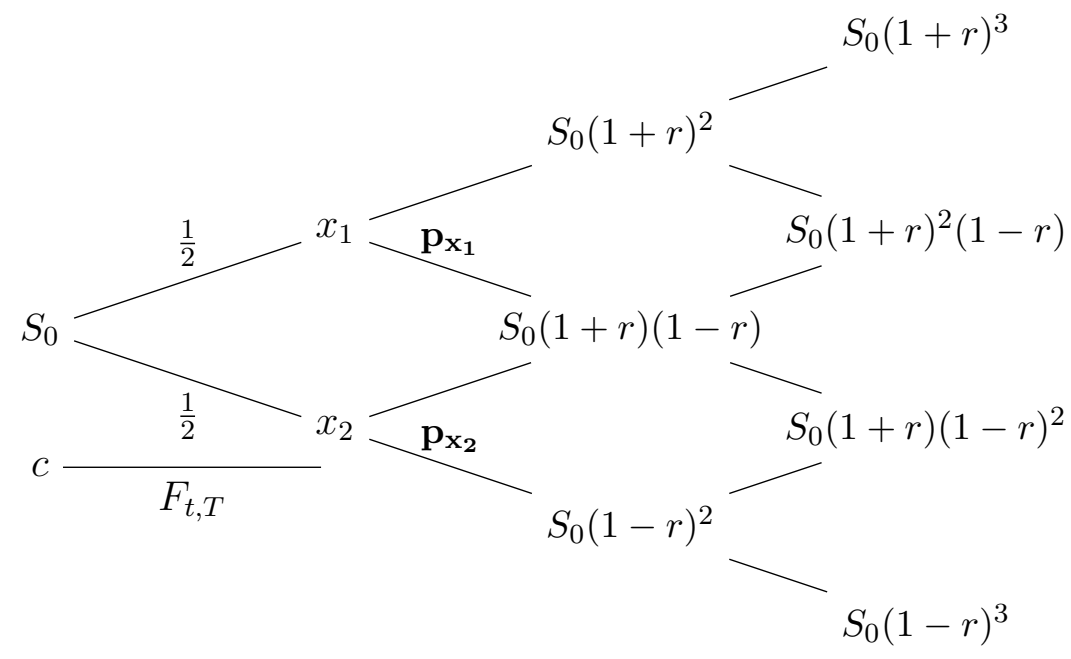

Figure 1: This is a discrete time representation of Example 2.1. In this figure $t^{*}=1$. The probability of going down after time 1 depends on whether the path goes through $x_{1}$ or $x_{2}$ at time 1. The futures price is constant and equal to $c$ between times 0 and 1 . 


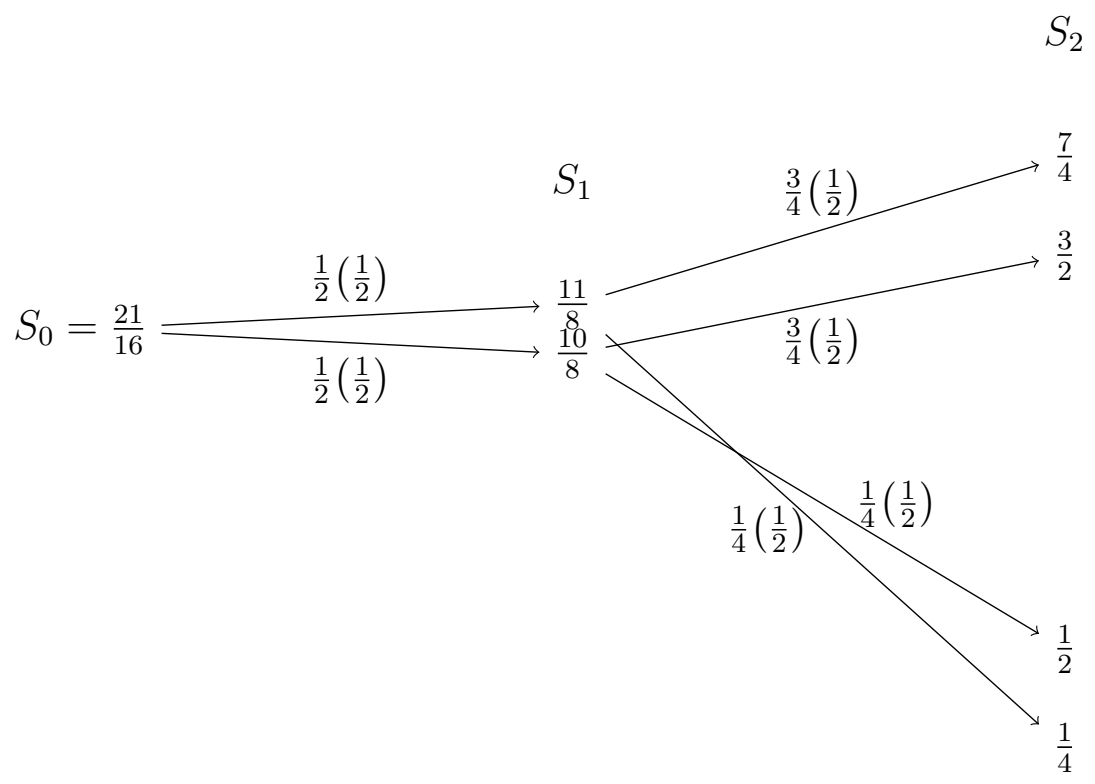

Figure 2: This is a discrete time representation of Example 5.5. We think of the $\omega_{i}^{\prime} s$ as the possible paths and the probabilities of going through each branch are written above the branch (the $Q^{*}$-probabilities being those in parentheses). 